Ueber die Eiweisskörper der Ricinussamen der Proteinkörner etc. 15

\title{
Ueber die Eiweisskörper der Ricinussamen der Proteinkörner, sowie der Krystalloide dieser Samen.
}

Von

\section{H. Rithausen.}

In der Schrift: Die Eiweisskörper der Getreidearten u. s. w., p. 182-187, (1872 erschienen) habe ich die Ergebnisse einer unvollendet gebliebenen Untersuchung iiber die Eiweissstoffe der Ricinussamen mitgetheilt. Da diese Samen grosse Mengen der als "Proteinkörner" und „Krystalloide" bekannten eiweissreichen Gebilde enthalten, so konnte die Untersuchung gleichzeitig iiber die Zusammensetzung und Eigensehaften der Eiweisssubstanzen derselben, ïber welche bis dahin keine eingehenderen chemisch-analytischen Arbeiten vorlagen, Aufschluss geben und iber deren Stellung zu den bis dahin näher untersuchten Körpern aufklären. Dies Ziel war, als die Arbeit abgebrochen werden musste, nicht erreicht; es hatten sich jedoch verschiedene, wie mir scheinen wollte, nicht unwichtige Thatsachen ergeben, die für die Beurtheilung der Körper sowohl, wie für den Gang der späteren Untersuchung von Bedeutung waren und die ich zunächst in Kürze mit den Worten, mit welchen ich sie bezeichnete, wieder anführen will.

Auf Seite 183 hob ich als vorläufiges Resultat bei Untersuchung der Krystalloide hervor:

1) „dass die Hauptmasse der Proteinsubstanz in den Krystalloiden nieht Legumin, sondern ein dem Eiweiss oder dem Gluten-Casein des Weizens nahestehender Körper sei;"

2) „dass aber ausser diesem noch andre, und zwar sehr leicht lösliche Eiweisskörper darin enthalten seien".

Ferner fand ich, p. 184 und p. 185, dass die vorläufig als Casein bezeichnete Masse aus der Lösung in Kaliwasser nur un. vollständig wieder gefällt werde, in Salzlösungen be- 
trächtlich löslich und somit ganz verschieden vom Legumin sei, schloss aber weiter aus der Untersuchung der Ca-Verbindung, auf ein Gemisch von Legumin and Eiweiss oder eines eiweissähnlichen Körpers und fasste das Gesammtergebniss in den Worten zusammen:

„Aus diesen noch sehr ungenügenden Untersuchungen geht mit einiger Sicherheit hervor, dass die Zusammensetzung der Krystalloide im Ricinussamen ziemlich complicirt ist und mindestens 2 verschiedene Proteinkörper, welche mit Legumin und Conglatin nicht identisch sind, darin vorkommen."

$\mathrm{Zu}$ diesen Schlussfolgerungen führten mich nicht nur die Beobachtungen bei Fällung aus schwach alkalischer Lösung durch Säuren (Essigsäure oder Schwefelsäure) und bei Darstellung der Kupfer-Verbindungen, sondern auch die analytischen Ergebnisse der gefällten Substanzen, der Kupferverbindungen und des durch Coagulation aus den Mutterlaugen erhaltenen Produkts. Nachdem ich nun in jüngster Zeit einige $N$-Bestimmungen, die früher sämmtlich mittelst Natronkalk -Verbrennung ansgeführt wurden, nach Dumas angestellt habe und hierbei zu wesentlich andern Resultaten gekommen bin, so kann die Vermuthung, dass Legumin einer der Bestandtheile der Ricinus-Krystalloide sei, als endgiiltig beseitigt bezeichnet werden; die sonstigen Schlussfolgerungen finden indess in den nachstehend mitzutheilenden Untersuchungen volle Bestätigung.

Die Analysen hatten bei N-Bestimmung 'mittelst Natronkalkverbrennung ergeben:

Im Mittel sämmtlicher Analysen und für aschefreie Substanzen

(Asche 2,38=3,23 Prc., Phosphorsäure 1,51-1,85 Prc.)

$\mathrm{C}=52,61$ Prc. für aschehaltige Substanzen $\mathrm{C}=51,26$ Prc.

$\mathrm{H}=7,05 \quad " \quad$ " $\quad$ " $\quad \mathrm{H}=6,87 \quad "$

$\mathrm{N}=16,93 \quad " \quad$ " $, \quad " \quad \mathrm{~N}=16,73$ "

$\mathrm{S}=0,96, \quad, \quad, \quad, \quad \mathrm{~S}=0,93$,

$0=22,45 \quad " \quad, \quad, \quad$ "

wogegen die volumetrische Bestimmung des N 18,00 (Rohprodukt) und 18,11; 18,19 Prc. ergiebt.

Beim Studium der Kupfer-Verbindungen erwies sich ein Theil der wie eben angegeben zusammengesetzten Substanz als sehr löslich in Salze enthaltendem Wasser und auch in reinem 
Wasser, so dass, je nachdem mit viel oder mit wenig Wasser ausgewaschen war, eine ganz verschiedene Zusammensetzung der in den Niederschlägen enthaltenen Proteinsubstanz, so wie auch eine grössere oder geringere Verminderung der Masse gefunden wurde. Ich theile hier die Zusammensetzung einiger solcher Kupferverbindungen, die kurze Zeit nach Veröffentlichung der a. a. O. mitgetheilten Resultate noch ermittelt worden war, mit, um damit die Richtigkeit der Beobachtungen zu erweisen.

1) Fällung aus der schwach alkalischen Lösung mit essigsaurem Kupfer; Reaction der Fällungsflüssigkeit sehr schwach, jedoch deutlich sauer; Niederschlag filtrirt, mit wenig Wasser gewaschen, danach mit grossen Mengen Weingeist von 40-50 Pre., zuletzt mit starkem Spiritus, mit absolutem Alkohol und über Schwefelsäure getrocknet. Zur Analyse bei $125^{0}$ getrocknet.

a) Cu-Verbindung.

I. II. III.

$\mathrm{C}=41,62 . \quad 40,58 . \quad 41,61$.

$\mathrm{H}=5,96 . \quad 5,60.5,51$.

$\left.\mathrm{N}^{1}\right)=13,41 . \quad 13,23 . \quad 13,44$.

$\mathrm{CuO}=18,07$. 20,18. 19,05.

Asche $=2,44 . \quad 2,20 . \quad 2,12$.

$\mathrm{S}=-\quad-0,75$.

b) Aus der Cu-Verbindung berechnete Zusammensetzung der Proteinsubstanz.

I. $\quad 1 \mathrm{I}$. III.

$$
\begin{array}{crr}
\mathrm{C}=52,30 . & 52,24 . & 52,78 . \\
\mathrm{H}=7,49 . & 7,20 . & 6,99 . \\
\mathrm{N}=16,87 . & 17,03 . \quad 16,97 . \\
& & \\
\mathrm{O} & 22,34 . \\
\mathrm{S} & =23,34 . \quad 23,53 . & 0,92 .
\end{array}
$$

Das Gewicht des trocknen Niederschlags entsprach nicht genau, aber annähernd der Menge der angewandten Proteinsubstanz, die sich vermindert hatte.

2) Fällung mit schwefelsaurem Kupfer; Reaction der Fällungsflüssigkeit sehr schwach sauer; Niederschlag mit Wasser ausgewaschen bis zum Verschwinden der Schwefelsäure-Reaction, wozu grosse Mengen erforderlich waren; hierauf mit Alkohol gewaschen und entwässert, dann über Schwefelsäure getrocknet.

a) Cu-Verbindung.

$$
\begin{aligned}
& \mathrm{C}=41,40 . \\
& \mathrm{H}=5,61 . \\
& \mathrm{N}=13,91 . \\
& \mathrm{S}=0,97 . \\
& \mathrm{CuO}=17,15 . \\
& \mathrm{Asch}=1,99 .
\end{aligned}
$$

b) Proteinsubstanz aus der Cu-Verbindung berechnet.

$$
\begin{aligned}
& \mathrm{C}=51,20 . \\
& \mathrm{H}=6,93 . \\
& \mathrm{N}=17,20 . \\
& \mathrm{O}=23,48 . \\
& \mathrm{S}=1,19 .
\end{aligned}
$$

1) Durch Natronkalkverbrennung bestimmt. 
Aus der Menge des $\mathrm{Cu}$-Niederschlags berechnete sich ein Verlust an Proteinsubstanz von 40-50.Pre; es gaben z. B. $100 \mathrm{gr}$ $67 \mathrm{gr}$ der Cu-Verbindung.

Die beträchtliche Abnahme im C-Gehalt der Substanz bei Auswaschen mit viel Wasser (52,61 Pre. zu 51,20, Abnahme: 1,41 Pre.) bewies, dass ein Gemenge vorlag von einem Creicheren und einem $\mathrm{C}$-ärmeren Proteinkörper.

Eine solche wesentlich C-reichere Substanz wurde beim Erhitzen der bei Fällung des Caseins aus sehwach alkalischer Lösung mittelst Säuren verbleibenden Mutterlaugen in namhafter Menge erhalten und deren Zusammensetzung aschefrei gefunden (a. a. 0. p. 186).
$\mathrm{C}=53,52 . \quad 53,11$.
$\left.\begin{array}{ll}\mathrm{H}=7,34 . & 7,41 . \\ \mathrm{N}=16,07 . & -\quad \text { (volumetrisch best.) }\end{array}\right\} \begin{gathered}\text { nahezu dem } \\ \text { Albumin. }\end{gathered}$

$\mathrm{Da}$ in diesen Mutterlaugen ausser der letzteren Substanz noch sehr erhebliche Mengen Proteinstoffe enthalten sind, die auch durch Coagulation in der Hitze nicht gefällt werden, wohl aber durch Kupfersalze unter Zusatz entsprechender Quantitäten Kalilösung, so geht auch hieraus, wie aus den Beobachtungen beim Wiederauflösen und Fällen der dargestellten Eiweisskörper und bei Darstellung der Cu-Verbindungen ganz klar hervor, dass die Ricinússamen resp. die Krystalloide und Proteinkörner Eiweiss körper in namhafter Quantität enthalten, die in verdünnten Salzlösungen leicht löslich sind, denn in allen Fällungsflüssigkeiten finden sich Salze: Kaliumsulfat, essigsaures Kalium, phosphorsaures Kalium u. s. w. Dasselbe habe ich für die Hülsenfruchtsamen, Lupinen und Mandeln schon längst dargethan ${ }^{1}$ ).

Auf die so gewonnenen Grundlagen stützen sich die nachfolgenden eingehenden Untersuchungen, bei welchen auch noch die Methoden berücksichtigt sind, welche von Andern zur Untersuchung von Proteinkörpern angewandt wurden, unter Andern von Sachsse und Weyl zur Untersuchung der Krystalloide der Paranuisse (Bertholletia).

1) Th. Weyl nimmt in seiner Abhandlung: Hoppe-Seyler, Zeitschrift Bd. I, 72-101 davon freilich keine Notiz; aber es mag sich unter manchen Umständen empfehlen, bereits bekannte oder nachgewiesene Beobachtungen und Thatsachen zu ignoriren. 


\section{Beschaffenheit des zur Untersuchung angewandten Materials.}

Während ich frïher zur Darstellung der Proteinstoffe eine Masse verwandte, die ich beim Absieben der grobgepulverten Pressrückstände von Ricinussamen durch ein feines Drahtsieb gewonnen hatte, diente zu den jetzigen Arbeiten eine Masse, die ich $\mathbf{d u r c h}$ Abschlämmen dieses ziemlich feinen Pulvers mit Aether erhielt. Das lockere, voluminöse Pulver wurde mit grossen Mengen Aether, in vielen kleinen Portionen nach einander angewandt, kräftig durchgeschüttelt und das im Aether suspendirte feine weisse Mehl mit dem Aether abgegossen, in dem es sich dann rasch zu Boden setzte. Das decantirte Krystalloidmehl (so mag die Masse bezeichnet sein) wurde nach dem Abgiessen des Aethers mit absolutem Alkohol gewaschen und kurze Zeit, um Wasserreste zu entfernen, damit in Berührung gelassen, hiernach uiber Sehwefelsäure bei gewöhnlicher Temperatur getrocknet, wobei es als feines weisses Pulver ohne Zusammenhang unter den einzelnen Theilchen verblieb.

Bei der mikroskopisehen Untersuchung konnten ausser den theilweise noch sehr gut erhaltenen Krystalloiden, die scheinbar reguläre Formen besassen und den Proteinkörnern, nur noch Zellenfasern in nicht erheblicher Menge, Schalenreste und eine sehr geringe Menge Oel nachgewiesen werden, womit die chemische Untersuchung vollkommen übereinstimmt.

Das zur Abschlemmung dienende Pulver enthielt nach p. 183 a. a. $0.8,74$ Pre. Stickstoff (Natronkalkbest.), einem Gehalt von 48,5 Pre. Proteinsubstanz entsprechend $\left(8,74 \cdot \frac{100}{18}\right), 16,48$ Pre. Fett und hatte bei der Behandlung mit Kaliwasser 32,0-32,4 Prc. trocknes Casein gegeben; es waren somit ca. 17 Pre. $\left.{ }^{1}\right)$ Proteinstoffe, wovon der in Kaliwasser unlösliche Rückstand geringe Mengen nur enthielt, in der Fällungsflüssigkeit gelöst geblieben.

In dem abgeschlemmten Krystalloidmehl dagegen fanden sich 12,48 Prc. N (Natronkalkbestimmung) für trockne Subst. $13,02, \mathrm{~N}$ (volumetrische Best.),

1) Oder auf $100 \mathrm{Th}$. der ursprünglichen Menge Proteinsubstanz: Gefällit: 66,4 Prc. Gelöst geblieben: 33,6 , 
entsprechend ca. 72 Pre. Proteinsubstanz, 0,43 Pre. Fett, 8,44 Prc. Asche und 16,7 in Kaliwasser unlöslicher Rïckstand, grösstentheils aus Fasern und Schalenresten bestehend, jedoch nicht frei von Stickstoff-Verbindungen.

Ich hoffte durch Auskneten dieses, in ein feines Leintuch gebundenen Mehls unter Alkohol den grössten Theil der Fasern u. s. w. abscheiden und eine fast reine Krystalloidmasse darstellen zu können; dies gelang indessen nicht, denn der N-Gehalt hatte sich nur auf 13,59 Pre. (volumetr.) erhöht und liess das Mikroskop noch zahlreiche feine Fasern und Schalenreste erkennen.

Beim Abschlemmen mit Aether war eine bedeutende Menge gröberen Pulvers zurückgeblieben, das noch sehr reich an Proteinsubstanzen, zur Benutzung bei den Untersuchungen aufbewahrt worden ist; obwohl es sich nach mehr als 5 jähriger Aufbewahrung sebr wenig von Schimmelpilzen durchzogen erwies, war die zersetzende Wirkung der sehr unerheblichen Pilzvegetation, wie die Versuche and das Mikroskop zeigten, doch von so geringer Bedeutung, dass es bei den Untersuchungen mit verwendet werden konnte. Das Mikroskop liess darin eine grosse Menge anscheinend freier, isolirter Krystalloide von grosser Regelmässigkeit der Form, - dem Ansehen nach Combinationen des regulären Octaëders oder Würfels, - und scharfen Contouren, so dass man wirkliche, vollkommen áusgebildete Krystalle vor sich zu haben glaubte, erkennen ${ }^{1}$ ); in dem Krystalloidmehl waren diese in verhältnissmässig viel geringerer Zahl vorhanden; ebenso zeigte die chemische Untersuchung einen beträchtlich höhern Gehalt an in warmem Wasser, so wie in den Chloriden von Kalium, Natrium, Ammonium, Calcium löslichen Proteinstoffen. Es enthielt im lufttr. Zustande 10,04 Pre. N (Natronkalkbest.), entsprechend einem Gehalte an Eiweisskörpern (mit 18 Prc. $\mathrm{N}$ ) = 55,77 Prc. - Bei der Analyse nach I (siehe später) gab es 32,3 Pre. durch Säure aus der Lösung in Kaliwasser, 14,91 Pre. durch CuLösung, im Ganzen 47,21 Pre. gefällte Proteinsubstanz, 28,72 in

1) Alle Proben zeigten, mit einem Tröpfchen Wasser durchrührt, bei 300 maliger Vergrösserung grosse Mengen solcher freiliegender Krystalloide; bei Verschiebung des Objektglases zählte ich in dem jedesmaligen Gesichtsfelde ca. 50-150 einzelne Krystalle. 
Kaliwasser unlöslichen Rückstand. Nicht fällbare N-Verbindungen enthielt es, als Proteinsubstanz berechnet, demnach ca. 8,5 Pre. Um Missdeutungen zu vermeiden babe ich die daraus dargestellten Substanzen besonders, als von $\mathbf{A}-\mathbf{R}$ stammend, bezeichnet.

\section{Untersuchungs methoden.}

Die verschiedenen zur Darstellung der Proteinsubstanzen angewandten Verfahren sind folgende:

I. Behandlung des Krystalloidmehl mit Kali-haltigem Wasser mit einem Gehalt von $1 / 2-1,5$ gr Kalihydrat p. Liter.

II. Behandlung desselben und der Masse A-R mit reinem Wasser von $40-50^{\circ} \mathrm{C}$.

III. Behandlung mit 10 pre. Kochsalzlösung.

IV. Behandlung mit $\mathrm{SO}_{4} \mathrm{H}_{2}$-haltigem Wasser und nachfolgender Extraction des Rückstandes mit Kali-Wasser.

V. Qualitative Untersuchungen iiber die Einwirkung verschiedener Salzlösungen und Säure-haltiger Wässer auf Krystalloidmehl und die Masse A-R.

I. In Kali-haltigem Wasser lösliche und durch Säuren fällbare Eiweisskörper des Krystalloidmehls.

Da ich zur Gewinnung der Proteinstoffe aus Hülsenfruchtund Oelsamen theilweise dies Kali-haltige Wasser als Lösungsmittel anwandte, so stelle ich die Untersuchung über die damit dargestellten Körper voran.

Wird Krystalloidmehl mit Kaliwasser, etwa 5 Prc. der angewandten Substanz an KHO bei der Verdünnung von $1 \mathrm{gr}$ per Liter enthaltend, übergossen, so wird bei gutem Durchriuhren der grösste Theil der Substanz in kürzester Zeit gelöst; es entsteht eine bräunlich gelbe, durch unlösliche Bestandtheile stark getriibte Lösung, die sich allmälig unter Abscheidung des Ungelösten als voluminöse Masse beinahe vollständig klärt, so dass sie nach etwa 8 bis 12 Stunden abgehoben und gefällt werden kann, der Riickstand wird filtrirt und ausgewaschen. Derselbe beträgt, zuletzt mit Spiritus gewaschen und dann über $\mathrm{SO}_{4} \mathrm{H}_{2}$ getrocknet, $16-18$ Pre. vom Gewicht der angewandten Substanz. Filtriren der frisch bereiteten trüben Lösung ergiebt sofort eine völlig klare Flüsssigkeit; da die Filtration aber schon nach wenigen Stunden fast ganz aufhört, so 
müssen die Filter öfters erneuert, und die verbrauchten, bei quantitativen Versuchen - für sich ausgewaschen werden. Die für diese Operationen geeigneten Temperaturen sind 5-8 ${ }^{\circ} \mathrm{C}$.

Die Lösungen werden mit verdünnter Essigsäure, am besten Schwefelsäure bis zur schwach sauren Reaction versetzt, wobei sich grosse Massen dichter käsiger und schnell zu Boden sinkender Flocken abscheiden. Der leicht zu filtrirende Niederschlag wird erst mit Wasser, dann mit grossen Mengen Spiritus von 70-80 Pre. gewaschen, hierauf die Masse in der Flasche mit absolutem Alkohol, Aether, zuletzt wieder mit Alkohol behandelt, dann ïber $\mathrm{SO}_{4} \mathrm{H}_{2}$ getrocknet.

Es verbleibt eine weisse etwas voluminöse und lockere in alkalischem Wasser leicht und klar lösliche pulvrige Substanz. Wasserhaltig getrocknet entstehen dagegen gelbbräunliche, hornartige, zähe, schwer za pulvernde Stücke. Für analytische Zwecke ist es vortheilhaft, die Masse nach Behandlung mit Aether mit wässrigem Weingeist befeuchtet über $\mathrm{SO}_{4} \mathrm{H}_{2}$ zu trocknen, um sie als dichte zusammengesinterte, hornartig durchscheinende, gelblichweisse Substanz zu erhalten. Die Löslichkeitsverhältnisse sind in allen Fällen dieselben.

Zur Ermittlung der Menge dieser Substanz wurden folgende Bestimmungen ausgeführt:

1.

2. 3.

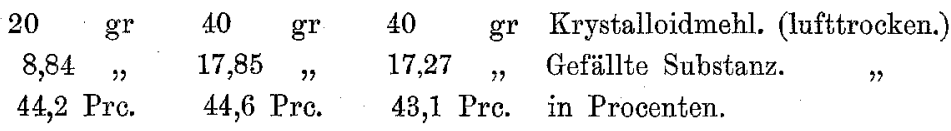

(Nahezu dieselben Mengen auf Trockensubstanz berechnet ${ }^{1}$ ).) Im Mittel demnach wurden 44 Pre. des Rohmaterials an Proteinsubstanz erhalten. Da dieses trocken nach der N-Analyse ca. 72 Pre. Eiweisskörper $\left(13,59 \cdot \frac{100}{18}\right)$ enthalten soll, so giebt dies Verfahren von 100 Proteinsubstanz nur 61 Pre. und bleiben 39 Prc. in der Fällungsflüssigkeit gelöst.

Zusammensetzung der gefällten Substanz (bei $130^{\circ}$ getr.)

$(s=$ Substanz; $\mathrm{V}=$ abgelesenes Vol. N-gas; $t=$ Temperatur; $\mathrm{B}=\mathrm{Ba}$ -

1) Krystalloidmehl 8,1 Prc., gefällte Substanz 8,4 Prc. Gewichtsverlust bei $125^{\circ}$. 
Ueber die Eiweisskörper der Ricinussamen, der Proteinkörper etc. 23

rometerstand in $\mathrm{mm} ; \mathrm{v}=$ corrig. Vol. $\mathrm{N} ; \mathrm{m}=$ Menge des $\mathrm{N}$. in Grm.; $\mathrm{P}=$ Procente $\mathrm{N}$ ).

1. 2. 3.4 4. $\quad$ 1. 2.4 .4 .4$.

$\mathrm{s}=0,2104.0,2375.0,2122.0,2083$.

$\mathrm{CO}_{2}=0,3953.0,4453.0,3979.0,3939 . \quad \mathrm{C}=51,24.51,14.51,14.51,14$ Prc. $\mathrm{H}_{2} \mathrm{O}=0,1321.0,1443.0,1360.0,1277 . \quad \mathrm{H}=6,97.6,75 . \quad 7,12.6,91 \quad "$

$\mathrm{N}$-Bestimmungen.

(Sämmtlich verschiedene Präparate.)

1.

$$
s=0,320
$$

$\mathrm{V}=49,2 \mathrm{ccm}$

$\mathrm{t}=15,0^{\circ}$

$\mathrm{B}=761,5$

$\mathrm{v}=45,76$

$\mathrm{m}=0,05747$

$p=17,96$ Pre.
2.

0,2095

$32,2 \mathrm{ccm}$

$14,8^{\circ}$

759,8

30,03

0,03772

18,00 Prc.
3.

0,2248

$3 \tilde{4}, 70 \mathrm{ccm}$

$15,6^{\circ}$

758,0

32,17

0,040405

17,97 Prc.

S-Bestimmung.

$\mathrm{s}=0,6956 ; \mathrm{BaSO}_{4}=0,0463 ; \mathrm{S}=0,0064=0,92$ Prc.

Asche-Bestimmungen.

$\mathrm{s}=0,6070 ;$ Asche: $0,0058=0,955$ Pro.

Hie rnach ist die Zusammensetzung:

$$
\begin{array}{lr}
\mathrm{C}=51,17 . & 51,66 \text { Prc. } \\
\mathrm{H}=6,91 . & 6,97 " \\
\mathrm{~N}=17,98 . & 18,15 " \\
\mathrm{~S}=0,92 . & 0,93 " \\
& 0=22,29 "
\end{array}
$$

Aschefrei.

Es wurde ferner eine Proteinsubstanz auf ihren StickstoffGehalt untersucht, die statt durch Schwefelsäure mit Kohlen$s$ äu gefällt war, und gefunden in 100 Theilen trockner Substanz Stickstoff:

$\mathrm{s}=0,4078 ; \mathrm{V}=64,00 \mathrm{ccm} ; \mathrm{t}=18,7^{\circ} ; \mathrm{B}=757,0 ; \mathrm{v}=58,16 ;$ $\mathrm{m}=0,07305 ; \mathrm{p}=17,937$ Prc.

Da nun 0,51 Pro. Asche gefunden wurden, so ist der Gehalt an $\mathrm{N}$ für aschefreie Substanz $=18,03$ Pre.

Bei dieser Fällung mit $\mathrm{CO}_{2}$ geben:

$1,7525 \mathrm{gr}$ Krystalloidenmehl $\quad 0,7014$ lufttr. Subst. $=40,2$ Prc.

5,00 2,003 $,=40,0$,

Auch eine durch Essigsäure bewirkte Fällung wurde auf C- und H-Gehalt untersucht und gefunden :

Aschefrei.

1. 2. 3. Mittel.

$\mathrm{C}=51,44 . \quad 51,15 . \quad 51,19 . \quad 51,26$.

$\mathrm{H}=6,84 . \quad 7,04 . \quad 6,80 . \quad 6,89$.

Asche $=1,66$ Prc.
2. 3. Mittel. $52,30 . \quad 52,01 . \quad 52,01 . \quad 52,12$. $6,95, \quad 7,19, \quad 6,95, \quad 7,03$. 
Die Fällung mit Essigsäure, welche immer nur mit wenig Wasser, dagegen mit grossen Mengen Weingeist gewaschen wurde, ist sonach reicher an $\mathrm{C}$ and da an Menge auch etwas mehr, als bei der Fällung mit Schwefelsäure gewonnen wird, weniger rein.

Bei der Auflösung des Krystalloidmehls in Kali-haltigem Wasser wurde nừ ein einziges Mal, als die Lösung vom Abend bis zum Morgen bei $8^{\circ} \mathrm{R}$. zur Klärung stehen blieb, beim Oeffnen des Gefässes $\mathrm{NH}_{3}$-Geruch beobachtet; da aber die gefällte Substanz dieselbe Menge N enthielt (s. oben N-Best., Analyse 2), als die übrigen, so ist sicher, dass dies $\mathrm{NH}_{3}$ nicht der dureh Säuren fällbaren Substanz angehörte, sondern den gelöst gebliebenen Eiweisskörpern, oder $\mathrm{NH}_{3}-V$ erbindungen, die in den Krystalloiden enthalten zu sein scheinen. Geruch nach $\mathrm{SH}_{2}$ wurde bei der Fällung einmal nur wahrgenommen und liess sich auch in diesem Falle beweisen, dass die gefällte Substanz intakt geblieben war.

Untersuchungen über die in den Fällungsflüssigkeiten enhaltenen Substanzen.

Diese Flïssigkeiten (einschliesslich der Waschwässer), welche an Salzen ausser eines Theils der in dem Krystalloidmehl enthaltenen Salze, auch die den angewandten Mengen $\mathrm{KHO}$ und $\mathrm{SO}_{4} \mathrm{H}_{2^{-}}$ oder Essigsäure entsprechende Quantität Kalisalze enthalten, geben beim Erhitzen bis nahe zum Kochen ein beträchtliches Coagulum; eine Analyse dieser Masse theilte ich oben (p. 18) mit. Um dies Erhitzen zu vermeiden, fällte ich die Proteinsubstanzen mittelst $\mathrm{CuSO}_{4}$ unter Zusatz von dem Cu-Salz entsprechenden Mengen Kalilösung aus, filtrirte den Niedersehlag, wusch ihn mit viel Weingeist, behandelte ihn darnach in Flaschen mit grösseren Portionen absoluten Alkohol, und trocknete zuletzt uiber $\mathrm{SO}_{4} \mathrm{H}_{2}$.

Die Quantität des Cu-Niederschlags betrug (lufttrocken):

$$
\begin{array}{cc}
\text { Von } 20 \text { gr } & \text { Von } 40 \text { gr Krystalloidmehl. } \\
6,349 " & 13,076 " \\
31,7 \text { Pre. } & 32,6 \text { Pre. }
\end{array}
$$

und wurde darin gefunden 13,74 Pre. Wasser (und Alkohol) und fuir die getrocknete Substanz 36,28 Pre. CuO und Asche, woraus sich an gefällter organischer Substanz berechnet: 
Ueber die Eiweisskörper der Ricinussamen, der Proteinkörner etc.

$$
\begin{array}{cccc}
\text { org. Substanz } & 3,49 \mathrm{gr} & 7,19 \mathrm{gr} \\
17,4 & \text { Pre. } & 17,9 \text { Pre. }
\end{array}
$$

Die C- und H-Bestimmung in dieser Masse ergab für die organische Substanz:

$$
\begin{aligned}
& \mathrm{C}=46,55 \text { Prc. } \\
& \mathrm{H}=6,06
\end{aligned}
$$

Aus dieser Cu-Fällung versuchte ich, nachdem sie zu feinem Pulver zerrieben, in sehr verdünnter Kalilauge wieder gelöst und wobei eine etwas triibe, nicht filtrirbare Lösung entstanden war, durch Zusatz von zur Lostrennung des $\mathrm{Cu}$ geniigenden Mengen verdïnnter Schwefelsäure reine Proteinsubstanz zu fällen, was auch theilweise gelang, jedoch bei der ǵrossen Löslichkeit der vorhandenen Eiweisskörper in schwefelsaurem Kali und verdünnter Schwefelsäure unter grossem Verlust. Ich erhielt einen voluminösen, flockigen weissen Niederschlag, der mit Wasser, Alkohol u. s. w. gewaschen und getrocknet (ïber $\left(\mathrm{SO}_{4} \mathrm{H}_{2}\right)$, ein weisses flockiges voluminöses Pulver bildete. 9,53 gr des Ca-Niederschlags gaben hiervon 2,046 gr, also 21,4 Prc., von der organischen Substanz $(4,76 \mathrm{gr})=100$, (bei einem Gehalt der Cu-Verbindung von zusammen 50,02 Pre. CuO, Asche und Wasser) = 43 Pre., sodass 57 Pre. gelöst blieben. Ihre Zusammensetzung fand ich in Bezug anf $\mathrm{C}$ - and N-Gehalt (C mit ehromsaurem Blei, N nach Dumas bestimmt) nach Trocknung bei $130^{\circ}$ und nach Abrechnung von 2,80 Pre. Asche

$$
\begin{aligned}
& \mathrm{N}=16,53 \text { Pre. ) (Nahezu die Zusammen- } \\
& \mathrm{C}=53,82, \% \quad\} \text { setzung des Glutenfibrins.) }
\end{aligned}
$$

Einige andre bei den ersten Untersuchungen schon dargestellte und in grösserer Menge erhaltene Niederschläge (s. „Die Eiweisskörper" p. 186) hatten bei damals schon ausgeführten Analysen gegeben ( $\mathrm{N}$ mit Natronkalkverbrennung bestimmt);

1. Cu-Terbindungen.

a. b.

$\mathrm{C}=41,43 . \quad 37,04$.

$\mathrm{H}=5,64 . \quad 5,12$.

$\mathrm{N}=13,32 . \quad 12,64$.

$\mathrm{CuO}$ u. Asche $=18,16 . \quad 23,16$.
2. Daraus berechnete org. Subst.:

a. b.

$50,62 . \quad 48,23$.

$6,89.6,67$.

16,27. 16,45 .

$0+\mathrm{s}=26,22 . \quad 28,65$.

b war, nachdem ich das beim Erhitzen entstandene Coagulum abfiltrirt und das Filtrat davon bis zu kleinen Volumen eingedampft hatte, durch $\mathrm{CuSO}_{4}$ und $\mathrm{KHO}$ gefällt worden.

Es geht aus diesen Untersuchungen unzweifelhaft hervor: 
1. dass in der Fällungsflüssigkeit erhebliche Mengen von Eiweisskörpern enthalten sind;

2. dass diese dureh Kupfersalze gefällt werden;

3. dass etwa 17,5 Prc., jedoch gemengt mit anderen Stoffen gefällt werden;

4. dass demnach von den N-haltigen, als Proteinstoffe mit 18 Pre. $\mathrm{N}$ berechneten 72 Pre. Substanzen, 44 Pre. durch Säuren, ca. 17,0 Prc. dureh Kupfersalze gefällt werden, während 11 Pre. noch gelöst bleiben;

5. dass die durch Cu-Salze fällbaren Eiweisssubstanzen eine grosse Löslichkeit in Salzlösungen, schwefelsaurem und essigsaurem Kalium, angesäuertem und alkalisehem, sowie auch reinem Wasser besitzen;

6. dass, da die Cu-Fällungen nicht reine Proteinsubstanz enthalten, auch die Zusammensetzung dieser letztern nicht genaü festzustellen ist.

Die $\mathrm{Cu}$-Fällungen verhalten sich uibrigens genan so, wie die Ci-Verbindnngen reiner Proteinkörper.

Untersuchung des Filtrats der Cu-Niederschläge.

Da die in Lösung verbliebenen N-Verbindungen nicht der Classe der Eiweisskörper, welche stets bis auf verschwindend kleine Mengen durch Cn-Salz und Kali gefälltt werden, angehören können, so musste versucht werden, die Natur derselben einigermaassen zu bestimmen, zugleich auch die Art der N-freien Bestandtheile der Ricinus-Krystalloide im Allgemeinen festzustellen.

Die nachfolgenden Angaben beruhen allein auf qualitativen Untersuchungen und ist in Folge dessen die Aufgabe als nicht vollständig gelöst zu betrachten.

Wird das Filtrat, das trotz schwach saurer Reaction wenig bläuliche Färbung zeigt, bis nahe zur Trockne im Wasserbade eingedampft, so tritt eine theilweise Reduction des nur in geringer Menge vorhandenen $\mathrm{CuO}$ ein und färbt sich der Rückstand gelb oder auch schmutzig grün. Wird dieser hierauf mit starkem Weingeist in der Wärme ausgezogen, so löst sich eine beträchtliche Menge Substanz unter bläulicher Färbung anf und verbleibt dieselbe nach Verdunstung des Weingeistes als gummiartige, auch nach langem Stehen über $\mathrm{SO}_{4} \mathrm{H}_{2}$ nicht erhärtende zähe Masse von schmutzig grüner Färbung (zufolge des Gehalts an $\mathrm{Cu}$ ). 
Dieser Rückstand, leicht löslich in Wasser und Weingeist, giebt:

1. mit Kalilauge gekocht $\mathrm{NH}_{3}$, ebenso

2. beim Glühen mit Natronkalk und in reichlicher Menge;

3. nach vorausgegangenem Kochen mit verdünnter $\mathrm{SH}_{2} \mathrm{O}_{4}$ die Trommer'sche Zuckerreaction, die ohne Säure nicht, oder erst nach längerem Kochen eintritt;

4. mit Cu-lösung and Kali entsteht eine tief blane klare Lösung;

5. Niederschläge entstehen nur mit ammoniakalischer Bleilösung und Quecksilberoxydnitrat.

Diese Reactionen lassen auf das Vorhandensein eines den Glycosiden ähnlichen Körpers, oder auf ein Gemenge einer Amid ähnlichen Substanz mit einem löslichen Kohlenhydrat schliessen. Krystallisation konnte ich nie beobachten, ebensowenig als die Ausscheidung einer krystallisirten Substanz aus der wässrigen oder alkoholischen Lösung. Leider ist mir eine etwas grössere Menge der Masse, ausreichend zu quantitativen Bestimmungen durch Unfall verloren gegangen.

Das rückständige Kaliumsulfat hinterlässt beim Auflösen geringe Mengen zäh flockiger Substanz; es entwickelt mit Alkalihydratlösung Ammoniak und enthält organische Stoffe, die sich als untrennbar rom Sulfat erwiesen.

Erwähnenswerth scheint mir noch, dass sich beim Verdampfen des Filtrats allmählig eine krystallinische Cu-Verbindung in geringer Menge zu Boden setzt und durch rechtzeitiges Abgiessen des Gelösten für sich gewonnen werden kann. Mit Natronkalk gemischt erhịtzt, giebt sie Ammoniak, sie verbrennt auf Platinblech unter starkem Erğlühen und lässt $\mathrm{CuO}$ als Rückstand. Näheres zu ermitteln war unmöglich.

Aehnliche Resultate erhielt ich bei Untersuchung eines von der Fällung des Caseins aus der Lösung von 5 gr Krystalloidmehl mit. $\mathrm{CO}_{2}$ gewonnenen Filtrats, das mit $\mathrm{SO}_{4} \mathrm{H}_{2}$ angesäuert worden war. Beim Eindampfen schied sich ziemlich viel flockige Proteinsubstanz ab (nach Gewicht nicht bestimmt); die davon filtrirte Fliissigkeit wurde concentrirt, mit Weingeist versetzt und längere Zeit zur Abscheidung des Kalisalzes damit bei gewöhnlicher Temperatur stehen gelassen und nach Entfernung der Lösung, mit noch mehreren Portionen Weingeist extrahirt, dann die gesammte Flïg- 
sigkeit mit absolutem Alkohol versetzt, welcher eine grössere Menge Substanz in dichten Flocken fällte. Das Gewicht derselben betrug 1,2 gr lufttrocken und gab sie bei einem Gehalt an Feuchtigkeit von 8,08 Pre., an Asche 28,00 Prc. (schwefels. Kalium), 10.31 Prc. N, aschefrei und trocken: 16,13 Pre. N. Die Menge dieser in Weingeist löslichen Proteinsubstanz von 5 gr Krystalloidmehl betrug hiernach $0,743 \mathrm{gr}=14,8$ Pre.

Da eine wässrige Lösung hiervon sämmtliche Eiweissreactionen gab, so ist hiermit wohl der Beweis geliefert, dass im Ricin.-Krystalloidmehl in Weingeist leicht lösliche Porteinkörper in nicht unbedeutender Menge rorkommen; ich bezeichnete diese als Kleber-Proteinstoffe oder Glutensubstanzen (wofür die Bezeichnung Glutenide gewählt werden könnte).

Die vor der Fällung filtrirte Lösung, liess nach dem Verdampfen einen Rückstand, der sich ganz wie der oben besprochene verhielt, nur dass er kupferfrei war.

II. Die in Wasser löslichen, so wie die Eiweisskörper des in Wasser unlöslichen Rückstandes.

R. Sachsse ${ }^{1}$ ) wandte zur Darstellung der Proteinsubstanzen der Krystalloide von Paranüissen die von Maschke zuerst versuchte Methode: Extraction mit Wasser von $40-50^{\circ} \mathrm{C}$. an und erhielt bei geeigneter Behandlung der Lösung eine in Form von Scheibchen sich ausscheidende Proteinsubstanz von der Zusammen-. setzung:

$$
\begin{gathered}
\mathrm{C}=51,00 ; \mathrm{H}=7,25 ; \mathrm{N}=18,06 ; \quad \mathrm{O}=21,51 ; \quad \mathrm{P}_{2} \mathrm{O}_{5}=0,82 ; \\
\mathrm{S}=1,36 .
\end{gathered}
$$

Ich erzielte bei dieser Behandlungsweise nicht sehr befriedigende Resultate, da sich beim Einleiten von $\mathrm{CO}_{2}$ in die Lösung nur geringe Mengen Proteinsubstanz und in unreinem Zustande abschieden, zum grösseren Theil gelöst blieb en. Bessere Resultate ergaben sich bei Anwendung des oben erwähnten Abschlemmungsrückstandes $A-R$. Eine sehr viel grössere Menge der Eiweisskörper des Krystalloidmehls bleibt, in Kaliwasser leicht löslich, im Rückstande der Behandlung mit Wasser.

Dass die Menge des aus der warmen wässrigen Lösung des

1) R. Sachsse, Die Farbstoffe, Kohlenhydrate und Proteinsubstanzen Leipzig bei Voss 1877. p, 315-316. 
Krystalloidmehls durch $\mathrm{CO}_{2}$ gefällten Proteinkörpers so gering war (2,5-3,2 Pre.), lässt sich auf verschiedene Weise erklären. Zuerst könnte als Grund hierfür angesehen werden, dass ich, um die in Wasser löslichen Substanzen möglichst vollständig zu entfernen, viel mehr Wasser, als Sachsse und Maschke anwandte, mithin sehr verdünnte Lösungen erhalten wurden; es ergab auch die von der $\mathrm{CO}_{2}$-Fällung filtrirte klare Flïssigkeit mit $\mathrm{Cu}$-Lösung und Kali erhebliche Niederschläge von Eiweiss-Kupferverbindungen.

Da das Krystalloid-haltige Material nicht von ganz frischen Samen, sondern von einem Pressriickstande stammte und es mit grossen Mengen Aether und zuletzt mit nicht grossen Mengen und nur für kurze Zeit mit absolutem Alkohol behandelt war, so könnte die geringe Löslichkeit der Eiweisssubstanzen durch theilweise Umwandlung in die coagulirte inodification herbeigeführt worden sein; dagegen spricht nur der Umstand, dass die Proteinstoffe des Rohmaterials und des mit Wasser erschöpften Rückstandes sich leicht und fast vollständig in Kaliwasser lösen und Alkohol nach den Beobachtungen von Pfeffer ${ }^{1}$ ) bei Ricin-Krystalloiden erst nach mehrwöchentlicher Berührung coagulirend wirkt.

Sonach kann auch angenommen werden, dass die Ricin-Krystalloide nicht von derselben Beschaffenheit und Zusammensetzung sind, wie die der Paranuss.

Die Untersuchung des an Krystalloiden sehr viel reicheren Abschlemmungsrïckstandes A-R, welcher eine sehr viel bessere Ausbeute an in Wasser oder Alkalichloriden löslicher und durch $\mathrm{CO}_{2}$ daraus fällbarer Substanz gab, beweist indessen, dass die Krystalloide des Krystalloidmehls durch Umbüllung von in Wasser überhaupt unlöslicher Proteinsubstanz vor der lösenden Wirkung des Wassers geschiutzt wurden.

\section{A. Die löslichen Substanzen des Krystalloidmehls.}

Nach etwa 1 stiundigem Digeriren bei $40^{\circ} \mathrm{C}$. wurde durch den Warmwassertrichter filtrirt, das Filtrat sogleich mit $\mathrm{CO}_{2}$ gefällt, der Niederschlag nach dem Abhitzen, nachdem die überstehende Fluissigkeit mittelst Heber abgezogen war, filtrirt, mit wenig Wasser, dann Alkohol und Aether gewaschen und hiernach iuber $\mathrm{SO}_{4} \mathrm{H}_{2}$ getrocknet.

1) Pringsheim, Jahrbücher für wissenschaftl. Botanik VIII ; p. 453. 
Er bildete eine weisse, bröckliche, körnige Masse, der ähnlich, die durch Wasser und $\mathrm{CO}_{2}$ aus Kochsalzlösung gefällt wird.

Es gaben

1) $20 \mathrm{gr}$ m.zusammen $1 \frac{1}{2}$ Lit. Wasser digerirt $0,652 \mathrm{gr}$ Subst. $=3,26$ Pre.

2) 20 " " $400 \mathrm{ccm}$ Wasser 0,492 " " =2,46 "

Die Analyse der Fällung beschränkte sich auf eine Bestimmung des $\mathrm{N}$ für bei $130^{\circ}$ getr. Subst.:

$$
\begin{aligned}
& \mathrm{s}=0,2375 ; \mathrm{V}=34,9 ; \mathrm{t}=19,2 ; \mathrm{B}=764,5 ; \mathrm{v}=32,09 ; \mathrm{m}=0,040205 ; \\
& \mathrm{p}=16,93 \text { Prc. }
\end{aligned}
$$

Sonach schien es, da die Substanz ziemlich unrein war, nicht von Bedeutung, grössere Mengen davon zu weiteren Analysen darzustellen.

Ausserdem wurden bei 1 durch Zusatz von 40 cem Kupferlösung allein $(0,8 \mathrm{gr} \mathrm{CuO}$ enthaltend $)$ gefällt: 1,301 gr, hiernach unter Zusatz von entsprechenden Mengen Kalilauge 3,689 gr CuVerbindung. Diese Niederschläge enthielten reichliche Quantitäten Proteinsubstanz, doch sind specielle Analy,sen nicht ausgeführt worden. Sie gaben beim Trocknen 13,0 Pre. Verlust; der CuOGehalt betrug für die gesammte Menge $0,8 \mathrm{gr}$; für beide Niederschläge berechnen sich daher 3,54 Pre. organische Substanz, grösstentheils Proteinsubstanz $=17,7$ Pre.

Das eingedampfte Filtrat dieser Cu-Niederschläge gab dieselben Reactionen, wie die gleichen Abdampfrickstände unter I.

B. Die löslichen Substanzen der Masse A - R.

65. gr gaben mit ca. 1,2 Liter Wasser 6,72 getrocknete Sub$\operatorname{stan} z=10,3$ Pre.

$$
\text { Analyse. }
$$

C- und H-Bestimmung.

1. 2

$\begin{array}{lll}\mathrm{s} & =0,2086 . & 0,1936 . \\ \mathrm{CO}_{2}=0,3967 . & 0,3663 . \\ \mathrm{H}_{2} \mathrm{O}=0,1261 . & 0,1152 . \\ \text { Asche }=0,0025 . & 0,0022 .\end{array}$
1. 2. Miftel.

$\mathrm{C}=51,87 . \quad 51,58 . \quad 51,72$.

$\mathrm{H}=6,72 . \quad 6,61 . \quad 6,66$. 
Ueber die Eiweisskörper der Ricinussamen, der Proteinkörner etc. 31

N-Bestimmung.

1. 2 . 2

$$
\begin{array}{cc}
\mathrm{s}=0,3206 . & 0,3025 . \\
\mathrm{v}=48,7 \mathrm{ccm} . & 45,95 . \\
\mathrm{t}=15,6^{\circ} . & 16,3^{\circ} . \\
\mathrm{B}=759,2 . & 766,0 . \\
\mathrm{v}=45,02 . & 42,77 . \\
\mathrm{m}=0,05655 . & 0,05369 .
\end{array}
$$

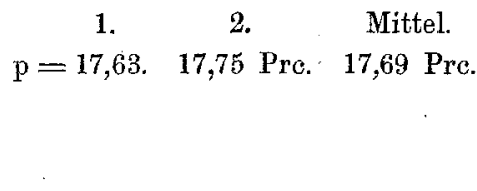

S-Bestimmung.

$\mathrm{s}=0,7605 ; \mathrm{BaSO}_{4}=0,0585 ; \mathrm{S}=0,00803=1,057$ Prc.

Hiernach ist die Zusammensetzung:

$\begin{array}{rrr} & \text { Aschefrei. } & \text { Substanz aus Bertholleti. } \\ \mathrm{C}=51,72 . & 52,34 . & 51,42 . \\ \mathrm{H}=6,66 . & 6,74 . & 7,31 . \\ \mathrm{N}=17,69 . & 17,90 . & 18,21 . \\ \mathrm{S}=1,06 . & 1,07 . & 1,37 . \\ \text { Asche }=1,19 . & 0=21,95 . & 21.69 .\end{array}$

Von der Zusammensetzung einer gleichen Substanz aus Bertholletia-Krystalloiden, nach der Analyse von Sachsse, weicht diese bedeutend ab, jedenfalls in Folge geringerer Reinheit.

Aus der Lösung in Kaliwasser wird die Substanz durch Säuren in sehr voluminösen Flocken unvollständig gefällt, bei einigem Ueberschuss an Säure, Schwefelsäure oder Essigsäure aber grösstentheils wieder gelöst. Mit Cu-Salz entstehen dieselben Fällungen, die Lösungen andrer Proteinstofie geben.

C. Die Proteinsubstanz der unlöslichen Rückstände von A und B.

Dieselbe wurde durch Kaliwasser, worin sie sich leicht, fast augenblicklich und vollständig löst, von den Schalenresten und Fasern getrennt, in derselben Weise, wie unter I angegeben ist und auch in derselben Weise behandelt.

Wasserhaltig und getrocknet gleicht sie ganz und gar der nach I. dargestellten Substanz.

Z u sammensetzung.

C- und H-Bestimmung.
a) Aus Krystalloidmehl.

$\begin{array}{lllll}1 . & 2 . & 3 . & 4 . & 5 .\end{array}$

$$
\begin{array}{lllllc}
\mathrm{s}=0,4813 . & 0,2344 . & 0,2222 . & 0,2623 . & 0,1773 . \\
\mathrm{CO}_{2}=0,8980 . & 0,4390 . & 0,4140 . & 0,4922 . & 0,3304 . \\
\mathrm{H}_{2} \mathrm{O}=0,2861 . & 0,1455 . & 0,1398 . & - & 0,1081 . \\
\text { Asche }=0,0061 . & 0,0020 . & 0,0024 . & 0,0029 . & -
\end{array}
$$




$\begin{array}{lcrrrrr} & 1 . & 2 . & 3 . & 4 . & 5 . & \text { Mittel. } \\ \mathrm{C}=50,89 . & 51,08 . & 50,81 . & 51,18 . & 50,90 . & 50,97 . \\ \mathrm{H} & =(6,69 ?) & 6,89 . & 6,99 . & - & (6,77 ?) & 6,94 . \\ \text { Asche }=1,27 . & 0,85 . & 1,08 . & 1,10 . & - & 1,08 .\end{array}$

N-Bestimmung.

1.2.

$$
\begin{array}{cc}
\mathrm{s}=0,3866 . & 0,3094 . \\
\mathrm{v}=61,0 . & 49,0 . \\
\mathrm{t}=\mathbf{1 8 , 5 .} & \mathbf{1 8 , 4 .} \\
\mathrm{B}=749,5 . & \mathbf{7 4 9 , 0} \\
\mathrm{v}=54,92 . & 44,11 . \\
\mathrm{m}=0,06898 . & 0,05540 .
\end{array}
$$

1. 2. Mittel. $\mathrm{p}=17,84 . \quad 17,906 . \quad 17,87$ Pre.

S-Bestimmung.

$\mathrm{s}=0,7581 ; \mathrm{BaSO}_{4}=0,042 ; \mathrm{S}=0,00576=0,76$ Prc.

Aschebest. $\mathrm{s}=0,5772 ;$ Asche $=0,0056=0,97$ Pre.

Hieraus ergiebt sich:

$$
\begin{aligned}
& \mathrm{C}=50,97 . \\
& \mathrm{H}=6,94 . \\
& \mathrm{N}=17,87 . \\
& \mathrm{S}=0,76 . \\
& \text { Asche }=1,025 .
\end{aligned}
$$

Aschefrei.<smiles>[131Sn]</smiles>

7,01 .

18,06 .

0,79 .

$0=22,65$.

b) Aus dem Abschlemmungsrückstande $A-R$.

C-Bestimmung.
1.
$2 . \quad 3$.
1. 2
3. Mittel.

$\mathrm{s}=0,2539 . \quad 0,2043 . \quad 0,1774 . \quad \mathrm{C}=50,86 . \quad 51,22 . \quad 51,026 . \quad 51,036$.

$\mathrm{CO}_{2}=0,4735 . \quad 0,3837 . \quad 0,3319 . \quad \mathrm{H}=(6,68 ?)(6,67 ?) \quad 6,95 . \quad 6,94$.

$\mathrm{H}_{2} \mathrm{O}=0,1527 . \quad 0,1238 . \quad 0,1113$.

N-Bestimmung.

$\mathrm{s}=0,2965 ; \quad \mathrm{V}=46,8 ; \quad \mathrm{t}=19,4 ; \quad \mathrm{B}=760 ; \quad \mathrm{v}=42,56 ; \quad \mathrm{m}=0,05346 ;$

$p=18,03$ Pre.

S-Bestimmung.

$\mathrm{s}=0,7044 ; \mathrm{BaSO}_{4}=0,045 ; \mathrm{S}=0,00615=0,87$ Prc.

Asche-Bestimmung.

$\mathrm{s}=0,5038 ;$ Asche $=0,0033=0,65$ Pre.

Die Zusammensetzung ist also:

$$
\begin{aligned}
& \mathrm{C}^{\prime}=51,036 . \\
& \mathrm{H}=6,94 . \\
& \mathrm{N}=18,03 . \\
& \mathrm{S}=0,87 . \\
& \text { Asche }=0,65 .
\end{aligned}
$$

Aschefrei.

51,37 .

6,99 .

18,14 .

0,87 .

$0=22,63$. 
Ueber die Eiweisskörper der Ricinussamen, der Proteinkörner etc. 33

Die oben p. 29 angewandten Mengen Krystalloidmehl gaben $1=20 \mathrm{gr} \quad . \quad 2=20 \mathrm{gr}$ $6,982 \mathrm{gr}=34,9$ Prc. $\quad 7,039 \mathrm{gr}=35,0$ Pre.

und die Masse $\mathrm{A}-\mathrm{R}=65 \mathrm{gr}: 8,93 \mathrm{gr}=13,7$ Pre.

Demnach war die Ausbeute an Proteinsubstanz:

Wässrige Lösung.

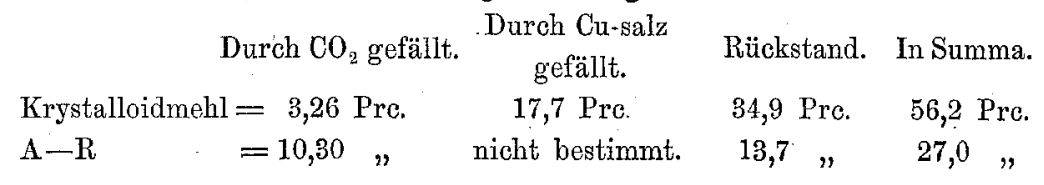

III. Die bei der Einwirkung von 10 Pre. NaCl-Lösung erhaltenen Proteinsubstanzen.

Th. Weyl ${ }^{1}$ ) wandte als auflösendes Mittel für die Krystalloide von Bertholletia diese Lösung an; aus der Lösung wird durch Zusatz von viel Wasser und darnach Einleiten von $\mathrm{CO}_{2}$ gefällt. Die Auszüge mit NaCl-Lösung zeigen saure Reaction. Die gefällte Substanz wird mit viel Alkohol und Aether gewaschen.

Ich habe das nämliche Verfahren auf das Krystalloidmehl und die Masse $A-R$ angewandt. Die Substanzen wurden mit NaCl-Lösung befenehtet im Mörser zerrieben, dann mehr NaClLösung zugesetzt; nach gutem Durchriihren filtrirte ich sogleich und wusch das Ungelöste mit derselben Flüssigkeit so lange, als im Ablauf beim Zusatz von Wasser noch deutliche Trübung eintrat; das gesammte Filtrat, das stets stark sauer reagirte, wurde mit viel Wasser verdünnt und dann $1 / 2-1$ Stunde hindurch $\mathrm{CO}_{2}$ eingeleitet; bei längerer Ruhe in der Kälte setzte sich die gefällte feinkörnige Substanz am Boden ab und wurde darnach die nicht völlig klare Lösung mit dem Heber abgezogen, der Bodensatz abfiltrirt, auf dem Filter mit nicht grossen Mengen Alkohol und Aether bei gewöhnlicher Temperatur gewaschen und ibber $\mathrm{SO}_{4} \mathrm{H}_{2}$ getrocknet. Sie bildet, wie die aus wässriger Lösung gefällte Substanz, eine weisse, bröckliche und feinkörnige Masse und giebt mit Wasser befeuchtet unter dem Mikroskop ein jener Substanz ähnliches Bild: sehr kleine rundliche, durchseheinende Körner oder Scheibchen.

Die Filtration der Lösung in $\mathrm{NaCl}$ geht bei diesen Massen sehr langsam vor sich und erfordert bei 10-20 gr Substanz 6 bis 10 Stunden, wenn man den" Rückstand möglichst erschöpfen will; 
ebenso setzt sich die durch Wasser und $\mathrm{CO}_{2}$ gefällte Substanz langsam nur vollständig ab, so dass ich bei den meisten Versuchen vom Abend bis zum Morgen stehen lassen musste. Die äusserst feinkörnige Fällung filtrirt anfänglich triibe.

Trocknet man die Fällung wasserhaltig, oder mit wässrigem Spiritus übergossen uiber $\mathrm{SO}_{4} \mathrm{H}_{2}$, so bleibt sie als gelblich-, oder grauweiss-hornartiger, durehscheinender, etwas spröder Rïekstand ohne jegliche Structur.

In der Salzmutterlange sind erhebliche Mengen Eiweisssubstanz gelöst, die ich durch Cu-Salz und Kali ausfällte. Im Extractionsrückstande bleiben ebenso grosse Mengen Eiweisskörper zurück, die ich dann durch Behandlung mit Kaliwasser vollständig gewann.

A. Die aus NaCl-Lösung gefäll te Substanz.

a) Krystalloidmehl.

$13 \mathrm{gr}$ gaben $1,195 \mathrm{gr} \mathrm{S} \mathrm{S}_{3}$-trockne Substanz. $=9,2$ Prc.

$10 " ; 0,993, ", \quad "=9,93$ ",

10 " mit $125 \mathrm{ccm}$ NaCl-Lösung nach und nach behandelt, $110 \mathrm{ccm}$

abfiltrirte Flüssigkeit mit $600 \mathrm{ccm}$ Wasser verdünnt, gaben 0,5 gr Substanz.

20 " mit $200 \mathrm{ccm}$ NaCl-Lösung in der Kälte digerirt, $120 \mathrm{cem} \mathrm{ab-}$ filtrirt; Rüickstand mit $200 \mathrm{ecm}$ Kochsalzlösung gewaschen, Filtrat $220 \mathrm{ccm} ; \mathrm{zu} 340 \mathrm{ccm}$ Filtrat 21/2 Liter Wasser; gab, $1,77 \mathrm{gr}=8,85 \mathrm{Prc}$.

C- und H-Bestimmung.

1. 2.

$\mathrm{s} \quad=0,2395 . \quad 0,2060$.

$\mathrm{CO}_{3}=0,4521 . \quad 0,3905$.

Analyse.

$\mathrm{H}_{2} \mathrm{O}=0,1461 . \quad 0,1253$.

$\mathrm{N}$-Bestimmung.

$\begin{array}{llll}1 . & 2 . & 3 . & 4 .\end{array}$

$\mathrm{s}=0,2188 . \quad 0,2606 . \quad 0,292 . \quad 0,247$.

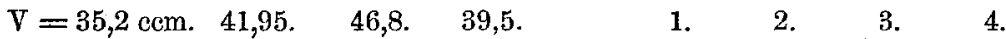

$\mathrm{t}=15,4^{\circ} . \quad 18,0^{\circ} . \quad 19,5^{\circ} . \quad 20,4^{\circ} . \quad \mathrm{p}=18,81 . \quad 18,54 . \quad 18,37 . \quad 18,36$.

$\mathrm{B}=760,5 . \quad 763,0 . \quad 763,0 . \quad 767,5 . \quad$ Mittel (unter Ausschluss von 1).

$\nabla=32,77 . \quad 38,47 . \quad 42,71 . \quad 36,11 . \quad \mathrm{p}=18,42$ Prc.

$\mathrm{m}=0,4116 . \quad 0,04832.0,05364.0,04535$.

Asche-Bestimmung.

$\mathrm{s}=0,4108 ;$ Asche $=0,0035=0,85$ Prc.

S-Bestimmung.

is $=0,6497 ; \mathrm{BaSO}_{4}=0,045 ; \mathrm{S}=0,00617=0,95$ Pre. 


\section{Zusammensetzung gefunden.}

$\begin{array}{lr} & \text { Aschefrei. } \\ \mathrm{C}=51,61 . & 52,05 . \\ \mathrm{H}=6,77 . & 6,83 . \\ \mathrm{N}=18,42 . & 18,57 . \\ \mathrm{S}=0,95 . & 0,96 . \\ \text { Asche }=0,85 . & 0=21,59 .\end{array}$

N-Bestimmung.

b) Abschlemmungs-Rückstand A-R.

1.

2.

$$
\begin{array}{lc}
\mathrm{s}=0,3032 . & 0,2026 . \\
\mathrm{v}=47,5 \mathrm{ccm} . & 31,7 . \\
\mathrm{t}=15,5^{\circ} . & 15,5^{\circ} . \\
\mathrm{B}=764,5 . & 763 . \\
\mathrm{v}=44,29 . & 29,62 . \\
\mathrm{m}=0,05563 . & 0,03720 .
\end{array}
$$

Aschebestimmung.

$\mathrm{s}=0,568 ;$ Asche $=0,0026=0,46$ Prc.

Demnach ist der $\mathrm{N}$-Gehalt fast genau derselbe wie der der vorigen Substanz.

C- und H-Bestimmung. Hierzu ein andres gleiches Präparat aus dieser Masse;

1-3 im Platinschiffchen im Luft- und Sauerstoffstrome, 4-6 mit chromsaurem Blei verbrannt.
1.
2.
3.
4.5.
6.

$\mathrm{s} \quad=0.2775 . \quad 0,2232 . \quad 0,2603 . \quad 0,2626 . \quad 0,2854 . \quad 0,2797$.

$\mathrm{CO}_{2}=0,5210 . \quad 0,4186 . \quad 0,4858 . \quad 0,4904.0,5337 . \quad 0,5268$.

$\mathrm{H}_{2} \mathrm{O}=0,1742$. 0,1358. 0,1692 . - $\quad$ - 0,1747 .

Mittel von

Asche $=$ - $\quad-\quad 0,0008$. $\quad-\quad$ - $\quad-\quad-\quad 1,2,5$ u. 6 .

$\mathrm{C}=51,21 \% .51,15 \% .50,9 \% .50,93 \% .51,03 \% .51,36 \% . \quad 51,19 \%$.

$\mathrm{H} \quad=6,97 \Rightarrow 6,76,7,22, \quad-\quad-\quad 6,94, \quad 6,89$,

Asche $=$ - $\quad$ - $0,30, \quad$ - $\quad$ - $\quad$ - $\quad 0,30$,

S-Bestimmung.

$\mathrm{s}=0,8444 \mathrm{gr} ; \mathrm{BaSO}_{4}=0,0600 ; \mathrm{s}=0,00823=0,97$ Prc.

Asche-Bestimmung.

$\mathrm{s}=0,5770 \mathrm{gr} ;$ Asche 0,0014 =0,24 Prc.

Die Zusammensetzung ist:

Aschefrei.

$$
\begin{aligned}
& \mathrm{C}=51,19=51,31 . \\
& \mathrm{H}=6,98=6,90 . \\
& \mathrm{N}=18,35=18,43 . \\
& \mathrm{S}=0,97=0,97 . \\
& \mathrm{O}=-\quad=22,39 . \\
& \text { Asche }=0,24 .
\end{aligned}
$$

(do. Subst, zur N-Best.) $=0,46$. 
Eine kleine Quantität dieses Körpers wurde in Kaliwasser gelöst und daraus durēh $\mathrm{SO}_{3}$ gefällt; es wurde nun folgende $\mathrm{Za}$ sammensetzung gefunden:

C- und H-Best. (1 im Platinschiffchen und 0-strome, 2 mit chroms. Blei verbrannt).

1.2.

$\mathrm{s}=0,2677 . \quad 0,2271$.

1. 2.

2.99

Mittel. $\quad$ Aschefrei.

$\mathrm{CO}_{2}=0,4992 . \quad 0,4246$.

$\mathrm{C}=50,86 . \quad 50,99$.

50,92 . 51,18 .

$\mathrm{H}_{3} \mathrm{O}=0,1636$. $\quad 0,1450$.

$\mathrm{H}=6,79 . \quad 7,09$.

6,94 . $\quad 6,96$.

Asche $=0,0014$.

Asche $=0,52$.

0,52 .

$\mathrm{S}=1,10$.

S-Bestimmung.

$$
\mathrm{s}=0,7737 ; \mathrm{BaSO}_{4}=0,063 ; \mathrm{S}=0,00864=1,10 \text { Prc. }
$$

$\mathrm{N}$-Bestimmung nicht ausgeführt.

Bei verschiedenen Bestimmungen der Menge des aus der Masse $A-R$ auf diese Weise erhaltenen Körpers ergab sich

$$
\begin{aligned}
& \text { 1) von } 20 \mathrm{gr}-3,642 \mathrm{gr}=18,2 \text { Prc. } \\
& \text { 2) } " 20 "-1,82,=14,1 \quad, \\
& 3) " 50 "-7,65 "=15,3 \quad,
\end{aligned}
$$

Bei 1 und 2 wurde auf je $20 \mathrm{gr} A-\mathrm{R}$ im Ganzen $350 \mathrm{ccm} 10$ pre. Kochsalzlösung verwandt und $300 \mathrm{ccm}$ filtrirte Flïssigkeit erhalten, die dann mit der vierfachen Menge Wasser verdünnt worden ist; in 3 wurden auf $50 \mathrm{gr}$, im Ganzen $500 \mathrm{ccm}$ Kochsalzlösung verwandt und 450 Filtrat erhalten.

Die gefällte Substanz erwies sich beim Auswaschen mit Wasser auf dem Filter, nachdem das Kochsalz grösstentheils entfernt war, als so löslich in Wasser, dass in 2 und 3 bedeutende Verluste eintraten; das Waschwasser schied, in Salzhaltige Flüssigkeit tropfend, eine Masse weissflockiger Substanz wieder $\mathrm{ab}$.

Die Ausbeute war also beinahe doppelt so gross als bei Krystalloidmehl.

$$
\begin{array}{cc}
\text { A-R. } & \text { Krystalloidmehl. } \\
\text { 18,2 Pre. } & 9,93 \text { Pre. }
\end{array}
$$

Aber auch in der Zusammensetzung der Substanzen beider Materialien zeigt sich ein wesentlicher Unterschied,

$$
\begin{array}{cc}
A-R . & \text { Krystalloidmehl. } \\
\mathrm{C}=51,31 . & 52,05 . \pm=0,71 . \\
\mathrm{H}=6,90 . & 6,83 . \\
\mathrm{N}=18,43 . & 18,57 . \pm=0,14 . \\
\mathrm{S}=0,97 . & 0,96 . \\
\mathrm{O}=22,39 . & \mathbf{2 1 , 5 9 .} \\
\text { Asche }=0,24 . & \mathbf{0 , 8 5 .}
\end{array}
$$


und darf aus der Differenz namentlich im Gehalt an $\mathrm{C}$ geschlossen werden, dass die Krystalloidreiche Masse A-R einen reinern Körper liefert, als das an Proteinkörnern und Globoiden reiche Krystalloidmehl.

Durch Auflösen in Kaliwasser und Fällen mit Säure aus dieser Lösung wird, wie die obige Analyse zeigt, die Zusammensetzung der Substanz uicht wesentlich geändert.

Zur Charakteristik der Substanz führe ich nur an:

1). dass sie frisch gefällt in warmem und in Wasser von gewöhnlicher Temperatur löslich ist, nach Behandlung mit Alkohol und Aether unlöslich;

2) dass sie frisch gefällt sich in $\mathrm{NaCl}$-Flüssigkeit wieder völlig löst, nach der Behandlung mit Alkohol und Aether nur theilweise ;

3) dass sie von Kaliwasser leicht und vollständig klar gelöst und aus dieser Lösung durch Säuren in sehr voluminösen, losen, langsam sich absetzenden Flocken unvollständig nur gefällt wird;

4) dass bei Ueberschuss von Säure in 3 keine Fällung, sondern eine weisslich truibe Flüssigkeit entsteht.

Wird die Zusammensetzung mit der von Weyl aus Bertholletia-Niissen dargestellten Substanz verglichen:

$\begin{array}{ccr} & \text { Ricinus. } & \text { Bertholletia. } \\ \mathrm{A}-\mathrm{R} . & \text { Krystalloidmehl. } & \\ \mathrm{C}=51,21 . & 52,05 . & 52,43 . \\ \mathrm{H}=6,90 . & 6,83 . & 7,12 . \\ \mathrm{N}=18,43 . & 18,57 . & 18,10 . \\ \mathrm{S}=0,97 . & 0,96 . & 0,55 . \\ \mathrm{O}=22,39 . & 21,59 . & 21,88 .\end{array}$

so ergeben sich wesentliche Unterschiede für den Gehalt an $\mathrm{C}$, $\mathrm{N}$ and $\mathrm{S}$.
B. Die aus der NaCl-Mutterlange durch Cu-Salze gefällten Substanzen.

Bei Zusatz von Cu-Lösung entsteht sofort ein beträchtlicher bläulich-flockiger Niederschlag, dessen Menge durch der angewandten $\mathrm{Cu}$-Lösung entsprechende Mengen Kalilauge vermehrt wird. 
Die Mutterlauge

a) Krystalloidmehl.

1) von $10 \mathrm{gr}$ gab bei sofortigem Zusatz der zur Zersetzung des Cu-Salzes erforderlichen Menge Kali 2,154 gr getr. Niederschlag $=21,5$ Prc.

2) von 20 gr gab desgl. $2,835, ", "=28,3$,

3) "20" " $" 4,694, " \quad " \quad=23,5 "$ Bei Bestimmung von $\mathrm{N}$, ferner $\mathrm{CuO}$ und Asche wurde in diesen Niederschlägen gefunden:

$\mathrm{CuO}$ und Asche: 23,93 Prc. ( $\mathrm{s}=0,9088$ bei $130^{\circ}$ getr.; $\mathrm{CuO}$ und Asche $=0,2175)$.

$\mathrm{X}$-Bestimmung.

$\mathrm{s}=0,5936 ; \quad \mathrm{V}=64,5 ; \mathrm{t}=15,6 ; \quad \mathrm{B}=756,5 ; \mathrm{v}=59,57 ; \mathrm{m}=0,07482 ;$

$\mathrm{p}=12,60$ Prc.,

woraus sich für reine Proteinsubstanz berechnet 16,56 Pro. N. Da die lufttr. gewogene $\mathrm{Cu}$-Fällung in 2) 2,835 gr betrug und 12,41 Pre. Trockenverlust, 23,93 Prc. Asehe und $\mathrm{CuO}$ gab, so waren darin 1,805 gr Proteinsubstanz enthalten, sodass 18,05 Pre. des Krystalloidmebls an Proteinsubstanz gefällt wurden.

b) Abschlemmungs-Riuckstand A-R.

1) Gefällt aus der Mutterlange von $40 \mathrm{gr}$ mit 100 .ecm $\mathrm{Cu}$ Lösung (2 gr $\mathrm{CuO}$ enthaltend) a. 6,262 und aus dem Filtrat hiervon dureh noch $40 \mathrm{ccm}$ Cu-Lösung. b. 2,97 gr tr. Substanz.:

Niederschlag a. enthielt 11,50 Prc. Feuchtigkeit u. 18,2 Prc. $\mathrm{CuO}$ u. Asche b. " $15,10 "$ "40,2 " " "

Als Gehalt an Eiweisssubstanz berechnet sich hieraus 5,79 $+1,31 \mathrm{gr}=7,10 \mathrm{gr}=17,75$ Pre.

Das Krystalloidmehl und die Masse A-R gaben also nahezu gleiche Mengen: 18,05 und 17,75 Prc. durch Cu-Lösung fällbare Eiweisssubstanzen.

Es besitzen diese Niederschläge sämmtlich das Verhalten und die Eigenschaften der $\mathrm{Cu}$-Verbindungen reiner Proteinkörper.

C. Die im Extractionsrückstande enthaltene Eiweisssubstanz.

Die Darstellung geschah in derselben Weise, wie in I. Erhalten wurde aus dem Riuckstande von 
Ueber die Eiweisskörper der Ricinussamen, der Proteinkörner etc. 39

1) $13 \mathrm{gr}$ Krystalloidmehl $3,30 \mathrm{gr}=25,4$ Prc.

2) $10 " \quad 2,235 n=22,35 n$

3) $10 " \quad 2,739 "=27,39$,

4) $20 " \quad$ " $" 274 n=26,37 "$

5) $20 " \quad$ " $\quad 5,063, n=25,06$, im Mittel $=25,30$ Prc.

lufttrockne Substanz.

Es gab somit die Krystalloidmasse:

1) direct mit Kaliwasser behandelt. 44,0 Pre.

Analyse.

C- und H-Bestimmung.

1.

$\mathrm{s} \quad=0,2239 . \quad 0,2370$.

$\mathrm{CO}_{\mathrm{z}}=0,4197$. $\quad 0,4448$.

$\mathrm{H}_{2} \mathrm{O}=0,1392 . \quad 0,1396$.
2) mit warmem Wasser extrahirt. 35,0 Pre.
3) mit NaCl-Lösung extrahirt. 25,3 Prc.

N-Bestimmung.

$\mathrm{s}=0,3656 ; \mathrm{V}=56,8 ; \mathrm{t}=20,1 ; \mathrm{B}=768,0 ; \mathrm{V}=52,03 ; \mathrm{m}=0,06535 ;$

$\mathrm{p}=17,87$ Prc.; eine 2. Best. gab in 0,2178: 0,03900 $\mathrm{N}=17,906$ Prc.

S-Bestimmung.

$\mathrm{s}=0,6825 ; \mathrm{BaSO}_{4}=0,0470 ; \mathrm{S}=0,00645=0,94$ Prc.

Asche-Bestimmung.

$\mathrm{s}=0,5135 ;$ Asche $=0,0057=1,11$ Prc.

Die Zusammensetzung ist hiernach.

$$
\begin{aligned}
& \mathrm{C}=51,16 . \\
& \mathrm{H}=6,91 . \\
& \mathrm{N}=17,89 . \\
& \mathrm{S}=0,94 . \\
& \text { Asche }=1,11 .
\end{aligned}
$$

A schefrei.

51,72 .

6,98 .

18,09 .

0,94 .

22,27 .

Der Körper gleicht in allen Eigenschaften der in $\mathrm{I}$ und II in derselben Weise dargestellten Substanz.

b) Abschl.-Riickstand A-R.

Der Extractionsriuckstand von $20 \mathrm{gr}$ gab:

$1,90 \mathrm{gr}$ mit $\mathrm{SO}_{3} \mathrm{H}_{2}$ gefällte Proteinsubstanz $=9,5$ Pre.

0,51 " "Cu-Lös. " " $\quad=2,55$,

im Ganzen 12,05 Prc. Proteinsubstanz.

In Bezug auf die Gesammtmenge der bei Behandlung mit Kochsalzlösung dargestellten, oder gefällten Proteinkörper ergiebt die Rechnung: 


\begin{abstract}
Krystalloidmehl. A-R.
Aus der Lösung durch Wasser und $\mathrm{CO}_{2}$ gefällt . . 10,00 18,2 Prc. Durch Cu-Salz ausgefällt . . . . . . . . . . 18,05 17,75 "

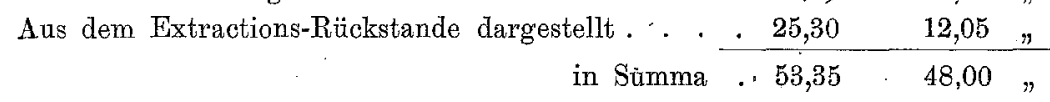

so dass von 72 Prc. Proteinsubstanz des Krystalloidmehls ca. 19 Prc. gelöst oder zum kleinen Theil ungelöst im Rückstande geblieben sind; die Menge dieses Riickstandes betrug bei $20 \mathrm{gr}$ Krystalloidmehl 4,62 $\mathrm{gr}=23,1$ Prc., wogegen bei der Behandlung I nur 16,7 Pre. rückständig waren. Die Masse A-R gab von $20 \mathrm{gr}$ 7,0 gr Unlösliches oder 35 Prc.

IV. Extraction des Krystalloidmehls mit verdünnter Säure and des Rückstandes hiervon mit Kalihaltigem Wasser.

Die Absicht war, zu ermitteln, ob die Extraction mit Säure enthaltendem Wasser, in welchem, wie Versuche zeigten, sich bedeutende Mengen Proteinsubstanz lösen, auf die Zusammensetzung der im Riickstande verbleibenden Eiweisskörper Einfluss hat. Ich wählte $\mathrm{SO}_{4} \mathrm{H}_{2}$-haltiges Wasser zu dem Versuch.

$10 \mathrm{gr}$ Krystalloidmehl wurde mit $500 \mathrm{cem}$ Wasser, dem $20 \mathrm{ccm}$ Schwefelsäure mit $0,8 \mathrm{gr} \mathrm{SO} \mathrm{SO}_{3}$ oder $0,98 \mathrm{SO}_{4} \mathrm{H}_{2}$ zugesetzt waren, 3 Stunden bei gewöhnlicher Temperatur unter häufigem Umschütteln digerirt, dann filtrirt, der Rückstand gewaschen und dieser hierauf mit $500 \mathrm{ccm}$ Wasser, vermischt mit $30 \mathrm{ccm}$ Kalilauge von 0,70 Gehalt an $\dot{\mathrm{K}}_{2} \mathrm{O}$ behandelt.

Die saure Lösung gab beim Neutralisiren mit Kali keinen Niederschlag, wurde daher sofort mit $45 \mathrm{ccm}$ Lösung von $\mathrm{CuSO}_{4}$ mit 0,9 $\mathrm{CuO}$ und entsprechender Menge Kali gefällt. Der Niederschlag wog lufttrocken $3,284 \mathrm{gr}$, gab Trockenverlust bei $130^{\circ}$ 14,86 Pre., Verbrennungs-Rückstand im getrockneten Zustande 37,28 Pre., enthielt daher 62,72 Pre. organisehe Substanz. Somit enthielten 3,284 gr 1,741 gr organische Substanz und wurden gefällt 17,41 Pre. des Krystalloidmehls.

$\mathrm{N}$ fand ich im Cu-Niederschlag.

$$
\begin{aligned}
& \mathrm{s}=0,3645 ; \mathrm{V}=29,2 ; \mathrm{t}=18,8^{\circ} ; \mathrm{B}=759 ; \mathrm{v}=26,48 ; \mathrm{m}=0,03426 ; \\
& \mathrm{p}=9,4 \text { Prc.; }
\end{aligned}
$$

für die organische Substanz berechnet sich daraus 15,07 Pre. N, bestand daher aus einem Gemisch N-haltiger und $\mathrm{N}$-freier Substanz, 
Proteinsubstanzen und Kohlehydraten. Es ist indessen die Existenz $\mathrm{N}$-ärmerer Verbindungen, als die Proteinstoffe, in den Krystalloiden sehr wahrscheinlich; so kann man auch, da Neutralisation der sauren Lösung keine Fällung bewirkte, diese als Hauptbestandtheile der Kupferfällung annehmen.

Aus dem Rückstande vom Schwefelsäure-Wasser wurden gewonnen $3,892 \mathrm{gr}$ Proteinsubstanz (lufttrocken), also 38,92 Pre. des Krystalloidmehls, und darin gefunden:

$\mathrm{N}$-Bestimmung.

$$
\begin{aligned}
& \mathrm{s}=0,3057 ; \quad \mathrm{V}=47,5 ; \mathrm{t}=19,2 ; \mathrm{B}=759,0 ; \quad \mathrm{O}=43,18 ; \mathrm{m}=0,05423 ; \\
& \mathrm{p}=17,74 \text { Pre. } \mathrm{N} .
\end{aligned}
$$

Asche: s $=0,6407 ;$ Asche $=0,0159=2,48$ Pre. ;

berechnet auf aschefreie Substanz 18,22 Prc. N.

Es geht daraus hervor, dass die Behandlung mit Schwefelsäurewasser in der Kälte die Löslichkeit des grössten Theils der Eiweisskörper in Kaliwasser nicht ändert, so dass diese in nicht sehr viel geringerer Menge, wie bei directer Behandlung mit Kaliwasser (44 Pre.), nur von etwas höherem $\mathrm{N}$-gehalt gewonnen werden.

Die Verhältnisse, in welchen die Bestandtheile des Krystalloidmehls bei den verschiedenen Behandlungsweisen abgeschieden, aus den Lösungen gefällt werden können, sind in übersichtlicher Zusammenstellung folgende:

$$
\text { I. II. III. IV. }
$$

$$
61 \text { Pre. 56,2. 553,3. 56,3 Pre. }
$$

und geht daraus hervor, dass im Krystalloidmehl grössere Mengen von Stoffen vorhanden sind, darunter N-haltige, die bei keinem dieser Verfahren gewonnen werden können und dass diese N-haltigen Körper nicht als Eiweisskörper angesehen werden dürfen. Die Menge der unfällbaren N-Verbindungen kann aus den Angaben der obigen Bestimmungen (IV) in Bezug auf $\mathrm{N}$-Gehalt leicht berechnet werden.

Auf Protein-

für 100 Krystalloidmehl. subst. mit 18 Prc. $\mathrm{N}$ ber.)

Gefällter Cu-Niederschlag . . 32,84 Prc. mit 2,61 Prc. N (= 14,48 Prc.) Gefällte reine Proteinsubst. (lufttr.) $38,92, \quad, 6,20, \quad \mathrm{~N}(=34,41 \%)$ in Summa . . . . . 8,81 Pre. $\mathrm{N}(=48,89$ ") In dem Krystalloidmehl, lufttr., enthalten . . 12,23 "N $(=67,87$ \#) Demnach $\mathrm{N}$ in leicht löslichen und nicht fällbaren Verbindungen (und in unlöslicher Verbindung) 3,42 Pro. N (=18,98 ") 
Ferner berechnet sich aus den Niederschlägen bei Behandlung mit $\mathrm{NaCl}$ (III):

Fällung aus der Lösung durch $\mathrm{CO}_{2}$ 9,93 Prc. mit 1,645 Prc. N (=9,13 Prc.)

Fällung aus der Lösung mit $\mathrm{Cu}-\mathrm{Salz} 28,35 \quad \# \quad, 3,150$ \# $\mathrm{N}(=16,48$ \#)

Substanz aus dem Rüickstande . 25,30 \#, 4,118, N $(=22,85 ")$

in Summa : . 8,913 \# $(=49,46$ ”)

Krystalloidmehl . . . . . . . . . . 12,23 " $\mathrm{N}(=67,87$ ")

Demnach nicht fällbarer $\mathrm{N}=3,317, \mathrm{~N}(=18,41$,

V. Qualitative Versuche über die Einwirkung verschiedener Salzlösungen auf Krystalloide.

Nachdem ich vor langer Zeit schon bei Untersuchungen über Legumin und Conglutin die Beobachtung gemacht hatte, dass in den Fällungsflüssigkeiten von diesen Körpern, die namhafte Mengen von Kalisalzen enthalten, beträchtliche Quantitäten von Eiweisskörpern gelöst bleiben, und die Existenz solcher in Salze enthaltenđen Flïssigkeiten löslichen Eiweisssubstanzen zur Genüge erwiesen war, ist dies auch für das Ricin-Krystalloidmehl durch die bereits mitgetheilten Untersuchungen wiederum dargethan worden und zwar für Flüssigkeiten, die schwefelsaures oder essigsaures Kalium neben Kaliumphosphat enthalten. Dennoch schienen mir einige Versuche wünschenswerth, ob Lösungen dieser Salze, sowie von Chlorkalium, weinsaurem Kalium u. a. bei directer Einwirkung auf die Substanz der Krystalloide, solche Materien daraus aufnehmen, resp. zu lösen vermögen.

Ich beschränkte meine Versuche auf die Salze:

schwefelsaures, phosphorsaures, weinsaures Kalium, essigsaures Natrium, Chlorkalium, Chlorammonium und Chlorcalcium,

und begnïgte mich mit dem Nachweis der Proteinsubstanz in den erhaltenen Lösungen, die ich stets nur bei gewöhnlicher Temperatur herstellte.

Die Proteinsubstanz wurde zumeist dadurch nachgewiesen, dass ich sie aus der klaren Lösung mit Cu-Salz und Kali fällte, den Niederschlag abfiltrirte und hierauf in Kalilauge löste, wobei die charakteristische violettblaue Färbung der Lösung entscheidend ist; es kann jedoch auch durch Säuren wieder gefällt werden.

Krystalloidmehl wurde mit Salzlösung befeuchtet, damit zèr- 
rieben bis zum gleichmässigen dicken Brei, hierauf von dieser noch etwas hinzugefügt und nach $1 / 2$ Stunde filtrirt.

Die Resultate sind kurz folgende:

a. Schwefelsaures Kalium, gesättigte Lösung, später durch Wasser verdünnt: be de utende Mengen gelöst.

b. Phosphorsaures Kalium (Triphosphat), 10 procentige Lösung; ebenso wie bei a.

c. Weinsaures Kalium: wenig gelöst.

d. Essigs. Natrium, 5 procentige Lösung: bedeuten de Mengen gelöst.

e. Chlorkalium, 12,5 procentige Lösung: be deutende Mengen gelöst; die Lösung trübte sich, wie eine mit $\mathrm{NaCl}$ bereitete, bei Zusatz von Wasser und setzte allmählich einen feinkörnigen Niederschlag ab.

f. Chlorammonium, 10 procentige Lösung: bedeutende Mengen gelöst; die Lösung trübte sich bei Zusatz von Wasser sehr stark, wie die mit KCl- nnd NaCl-Lösung bereitete und setzte wie diese einen feinkörnigen Niederschlag ab.

g. Chlorealcium, ea. $10 \mathrm{Pre}$. $\mathrm{CaCl}_{2}$ enthaltende Lösung, löste ebenso beträchtliche Men gen und verhielt sich die Flüssigkeit bei Zusatz von Wasser genau so, wie die mit $\mathrm{NaCl}$, $\mathrm{KCl}, \mathrm{NH}_{4} \mathrm{Cl}$ bereiteten Flüssigkeiten.

Keine der in $a-d$ erhaltenen Lösungen zeigte ein Verhalten gleich dem von $\mathrm{KCl}, \mathrm{NaCl}, \mathrm{NH}_{4} \mathrm{Cl}$ und $\mathrm{CaCl}_{2}$, sie blieben bei Zusatz von Wasser völlig klar, obwohl in allen, mit Ausnahme der von weinsaurem Kalium, Proteinsubstanzen in grosser Menge gefunden wurden.

Wenn nun auch daraus, dass sich in den genannten Salzlösungen ein grosser Theil der in den Krystalloiden enthaltenen Proteinstoffe löst, nicht ohne Weiteres auf ein Lösungsvermögen der Salze geschlossen werden kann, weil dem Wasser allein schon ein solches angehört, so lässt es sich doch auf Grund der von mir ausgeführten mannichfachen Versuche ungezwungen folgern und drängen verschiedene Beobachtungen, wie an den betreffenden Orten erwähnt wurde, zur Annahme desselben. Es hängt dies Vermögen aber von der besondern Natur and Beschaffenheit der Eiweisskörper ab.

Da die Wirkung des Chlorkaliums, Chlorammoniums und Chlorealciums dieselbe wiediedes Kochsalzes ist, 
wird die Meinung nahegelegt, dass die Lösungsfähigkeit dieser Chlor-Verbindungen darauf beruhen möge, dass sie durch die Phosphorsäure der Proteinkörper eine theilweise Zersetzung erleiden, Salzsäure gebildet werde, welehe sich mit bestimmten Eiweisskörpern bei Gegenwart von Chloriden zu leicht. löslicher Verbindung, die durch viel Wasser unter Abscheidung eines Theils des Eiweisskörpers wieder zersetzt wird, vereinigt.

Schon von Bouchardat') ist die lösende Wirkung höchst verdünnter Salzsäure oder besser eines mit wenig Salzsäure versetzten Wasser (z. B. 1 ccm Säure auf 1 Liter Wasser) auf Weizenkleber erwiesen worden, eine Beobachtung, die ich bei meinen Untersuchungen des Klebers als volkommen begründet und richtig erkannte ${ }^{2}$ ); es kann so kein Zweifel dariber bestehen, dass höchst geringe Mengen Salzsäure schon im Stande sind, einige an sich wenig lösliche Proteinstoffe löslich zu machen und eine Wirkung herbeizufübren, wie sie für $\mathrm{KCl}-, \mathrm{NaCl}-\mathrm{NH}_{4} \mathrm{Cl}-$ und $\mathrm{CaCl}_{2}-$ Lösung auf Krystalloide besteht, bei welcher letztern allerdings noch die Gegenwart eines grossen Ueberschusses an Chloriden nothwendig erscheint.

Ich untersuchte zu weiterer Aufklärung die Einwirkung eines solchen Salzsäure-haltigen Wassers auf das Ricin-Krystalloidmehl and bestätigte, freilich nur qualitativ, dass davon grössere Mengen Proteinsubstanz in der Kälte gelöst wurden; bei Gegenwart grösserer Mengen von Alkali-Chloriden wird das Lösungsvermögen insofern gesteigert, als diese die zersetzende Wirkung des Wassers auf die ClH-Eiweisskörper theilweise wenigstens aufheben.

Schwefelsäure-Wasser wirkte, wie nachgewiesen worden ist, ähnlich dem ClH-Wasser, jedoch schwächer und wurden gegen 14 Prc. Substanz weniger als von 10 Pre. NaCl-Flüssigkeit gelöst; dasselbe beobachtete ich für Essigsäure-Wasser.

Da KCl bei der Ernährung der Pflanze, nach den überzengenden Versuchen von $\mathrm{N}_{0 b b e^{3}}{ }^{\text {) }}$ eine bedeutende Rolle spielt, nicht aber $\mathrm{NaCl}$, das ja immer nur in änsserst geringen Mengen in

1) Compt. rend. de 1'Acad. XIV, 962.

2) Die Eiweisskörper der Getreidearten u. s. w. p. 25.

3) Die landwirthschaftlichen Versuchsstationen. Bd. 13. 
Ueber die Eiweisskörper đer Ricinussamen, der Proteinkörner etc. ${ }_{4} 45$

Pflanzen vorkommt, so kann die eben bezeichnete Wirkung desselben auf Proteinstoffe zur Aufklärung der Rolle des Chlorkaliums bei der Pflanzenernährung mit beitragen.

Die Resultate vorstehender Untersuchung will ich nun in Folgendem kurz zusammenfassen.

1) Die Proteinkörner und Krystalloide der Ricinussamen enthalten ausser Eiweisskörpern andere N-haltige Verbindungen, leicht löslich in Wasser und Salze-haltigem Wasser, wahrscheinlich als Glycoside; jedenfalls sind diese Verbindungen, welcher Art sie auch sein mögen, wesentlich ärmer an $\mathrm{N}$, als die Eiweisskörper und wie es scheint, nicht krystallisirbar:

2) Da alle Lösungen vom Krystalloidmehl nach Abscheidung der Eiweisskörper Reactionen auf Traubenzucker geben, wie R. Sachsse bereits für Bertholletia-Proteinkörner gefunden hat, so muss dieser als ein Bestandtheil der Proteinkörner ángenommen werden, obwohl diese Glycose auch als aus der Zersetzung von Glycosid-artigen Verbindungen hervorgegangen angesehen werden kann.

3) Wasser von $40-50^{\circ}$ C. löst aus diesem Mehl Proteinsubstanzen, in einiger und in um so grösserer Menge auf, je reicher die Substanz an freien, isolirten Krystalloiden ist, und folgt daraus, dass die Masse der Krystalloide darin grösstentheils löslich, die Masse der Proteinkörner und der Hüilmasse der Krystalloide fast ganz unlöslich ist.

4) Aus dieser wässrigen Lösung wird durch $\mathrm{CO}_{2}$ ein Theil der gelösten Eiweisskörper in kleinen Körnchen oder Scheibchen gefällt, ein anderer grösserer Theil bleibt gelöst; es gab die Lösung von

$$
\begin{array}{cc}
\text { Krystalloidmehl. } & \text { Masse A-R. } \\
\text { 3,2 Pre. } & \text { 10,30 Pre. }
\end{array}
$$

an gefällter Substanz.

Dieselbe ist ihrer Zusammensetzung nach nicht rein und nur ein Präparat nähert sich in der Zusammensetzung der, welche Sachsse für die aus Paranïssen dargestellte Substanz ermittelt hat, während es dem von Weyl aus Bertholletia-Krystalloiden mittelst NaCl-Lösung erhaltenen einige Aehnlichkeit zeigt. 
Ricinus.

$\begin{array}{lrr} & \text { Weyl. } & \text { Sachsse. } \\ \mathrm{C}=52,34 . & 52,43 . & 51,42 . \\ \mathrm{H}=6,74 . & 7,12 . & 7,31 . \\ \mathrm{N}=17,90 . & 18,10 . & 18,21 . \\ \mathrm{S}=1,07 . & 0,55 . & 1,37 . \\ \mathrm{O}=21,95 . & 21,88 . & 21,69 .\end{array}$

5) NaCl-Lösung (10 Prc.), löst gleichfalls bedeutende Mengen Eiweisskörper und zwar um so mehr, je reicher die Masse an Krystalloiden ist; so dass die Substanz der Krystalloide als grösstentheils löslich, die.Masse ihrer. Hüllsubstanz und der Proteinkörner als zum grösseren Theil unlöslich darin erscheint.

6) Wasser und $\mathrm{CO}_{2}$ fällen aus der durch die genannten Chloride bewirkten Lösung nur einen Theil der Eiweisskörper áls weisse körnige, oder Scheibchen-artige Masse, während eine beinahe ebenso grosse oder grössere Menge in der Mutterlauge gelöst bleibt ${ }^{1}$ ).

Gelöst im Ganzen . . . . . . $\begin{array}{cc}\text { Krystalloidmehl. } & \text { Masse A-R Prc. } \\ & 36 \text { Prc. }\end{array}$

Davon gefällt durch $\mathrm{H}_{2} \mathrm{O}$ und $\mathrm{CO}_{2}$. 9,93 " 18,2,

7) Der gefällte Proteinkörper ist bezüglich des Gehalts an C, $\mathrm{N}$ und $\mathrm{S}$ wesentlich versehieden, nicht nur von der aus Bertholletia-Krystalloiden von Weyl dargestellten, es sind auch die aus dem Krystalloid-reichen Abschlemmungsrïckstande und aus Krystalloidmehl erhaltenen Körper im Gehalt an $\mathrm{C}$ wesentlich von einander versehieden.

Ricinus.

Bertholletia.

A-R. Krystalloid-Mehl.

$\begin{array}{rrr}\mathrm{C}=51,31 . & 52,05 . & 52,43 . \\ \mathrm{H}=6,90 . & 6,83 . & 7,12 . \\ \mathrm{N}=18,43 . & 18,57 . & 18,10 . \\ \mathrm{S}=0,97 . & 0,96 . & 0,55 . \\ \mathrm{O}=22,39 . & 21,59 . & 21,88 .\end{array}$

Ebenso zeigen die Aschengehalte beträchtliche Unterschiede:

Ricinus.

Bertholletia.

A-R. Krystalloidmehl.

0,24 Prc. 0,85 Prc. 2,66-5,36 Prc.

1) Aus den Mittheilungen von Weyl über die Bertholletia-Krystalloide (Hoppe-Seyler, Zeitschr. I, 72-101) ist nicht ersichtlich, wie gross die Menge der gefällten Substanz im Verhältniss zum Rohmaterial ist. 
Hiernach darf man schliessen, dass, je reicher die angewandte Substanz an Krystalloiden ist, der Gehalt des aus dieser Lösung gefällten Proteinkörpers an $\mathrm{C}$ und Ascbe, um so mehr sich erniedrigt. Das Präparat aus $A-R$ ist beinahe frei von Aschensubstánzen.

8) Die Zusammensetzung des Körpers aus Krystalloidmehl weicht von der des Conglutins aus gelben Lupinen im C-gehalt noch erheblich ab, wogegen das Präparat aus $\mathrm{A}-\mathrm{R}$ diesen sehr nahe kommt.

\begin{tabular}{ccc} 
Conglutin & \multicolumn{2}{c}{ Substanz aus } \\
aus gelben Lupinen. & A-R. & Krystalloid-Mehl. \\
$\mathrm{C}=50,83$. & $51,31$. & $52,05$. \\
$\mathrm{H}=6,92$. & $6,90$. & $6,83$. \\
$\mathrm{N}=18,33$. & $18,43$. & $18,57$. \\
$\mathrm{S}=0,91$. & $0,97$. & $0,96$. \\
$\mathrm{O}=23,04$. & $22,39$. & $21,59$.
\end{tabular}

9) Ausser dem Kochsalz lösen auch $\mathrm{KCl}, \mathrm{NH}_{4} \mathrm{Cl}$ und $\mathrm{CaCl}_{2}$ in etwa 10-12 Pre. Lösung grosse Mengen Eiweisssubstanz auf und verhalten sich die entstehenden Flüssigkeiten gegen Wasser und $\mathrm{CO}_{2}$ genau wie die von NaCl-Lösung.

Ebenso wird von Lösungen des sehwefelsauren, weinsauren, phosphorsauren Kaliums, des essigsauren Natriums und wahrscheinlich noch mancher andern Salze Eiweisssubstanz in namhafter Menge gelöst, durch Wasser und $\mathrm{CO}_{2}$ jedoch keine Fällung bewirkt.

10) Saure, mit wenig Salz-, Schwefel-, Essigsäure gemischte Wässer, haben eine den zuletzt genannten Salzen ähnliche Wirkung.

11) Wasser mit 1 gr KHO pr. Liter löst fast die gesammte Menge der Eiweisskörper des Krystalloidmehls und des Rückstandes A-R augenblicklich auf, ebenso der Extractionsrickstände von der Behandlung mit Wasser, Salzen und Säuren.

Es gaben die verschiedenen hierzu angewandten Materien folgende Mengen:

A) Fällung aus der Lösung in Kaliwasser durch Säuren:

a) Kryst.-Mehl. b) $\mathrm{A}-\mathrm{R}$. c) Wasser-Extract. d) NaCl-Extr. e) $\mathrm{SO}_{3}$-Extr.

Rückst. v. Kryst.-Mehl. Rückst. von Rückst. von

$$
44,0 ; \quad 32,3 ; \quad 34,9 ; \quad 25,30 ; \quad 9,5 ; \quad 38,9 \text { Pre. }
$$

B) Durọh Cu-Salz und auf andere Weise gefällte Substanzen:

\begin{tabular}{rrrrrr} 
& 17,$0 ;$ & 14,$9 ;$ & 21,$3 ;$ & 28,$00 ; 38,5 ;$ & 17,4 Prc. \\
\hline in $\mathrm{Sa}_{;}^{\mathrm{a}} 61,0 ;$ & 47,$2 ;$ & 56,$2 ;$ & 53,$30 ; 48,0 ;$ & 56,3 Prc.
\end{tabular}


Da das Krystalloidmehl bei Berechnung des gefundenen $\mathrm{N}$ auf Eiweisskörper mit 18 Pre. N, nach p. 19 und 20 ca. 72, lufttrocken 67,87 Pre. Eiweisssubstanzen enthielt, die Masse A-R nach pag. 20 55,77 Prc., so ergiebt die Vergleichung dieser Zahlen an nicht fällbaren N-Verbindungen (als Eiweisskörper mit 18 Pre. $\mathrm{N}$ berechnet) :

6,87 Pre. 8,5 Prc. 11,67 Prc. 14,47 Prc. 7,77 Prc. 11,57 Prc. $\left.{ }^{1}\right)$.

12) Die mittelst Kaliwasser gelösten und durch Säure gefällten Substanzen haben eine nahezu übereinstimmende Zusammensetzung.

1) Kryst.-Mehl direkt mit

KHO-Wasser behandelt.
2) Wasser-Extract.-

Rückst.
3) NaCl-Extr.-

Rüickst.
4) $\mathrm{SO}_{3}$-Extr:Rückst:

a) mit $\mathrm{SO}_{3}$. b) mit $\mathrm{CO}_{2}$. c) mit Essigs. Kryst.-Mehl. $\mathrm{A}-\mathrm{R}$. mit $\mathrm{SO}_{3}$ gef: mit $\mathrm{SO}_{3}$ gef.

\begin{tabular}{rcrrrrr} 
& \multicolumn{7}{c}{ gefällt. } & mit $\mathrm{SO}_{3}$ gefällt. & \\
$\mathrm{C}=51,66$. & - & $52,17$. & $51,49$. & $51,37$. & $51,72$. &. \\
$\mathrm{H}=6,97$. & - & $7,03$. & $7,01$. & $6,99$. & $6,98$. & - \\
$\mathrm{N}=18,15$. & $18,03$. & - & $18,06$. & $18,14$. & $18,09$. & $18,22$. \\
$\mathrm{S}=0,93$. & - & - & $0,79$. & $0,87$. & $0,94$. & - \\
$\mathrm{O}=22,29$. & - & - & $22,65$. & $22,63$. & $22,27$. & -
\end{tabular}

13) Die bei directer Behandlung des Krystalloidmehls mit Kali-Wasser gelöste und durch Säure gefällte Proteinsubstanz enthält grössere oder geringere Mengen der auch in Wasser, Salzlösungen und angesäuertem Wasser löslichen Eiweisskörper, die im isolirten Zustande sich leicht in Kaliwasser lösen und durch Säure flockig, jedoch unvollständig gefällt werden.

14) Dieselbe verhält sich wie ein Gemenge von ähnlichen Körpern, die in Bezug auf Löslichkeit in reinem, Salze- oder Säurehaltigem Wasser versehieden und auch in der Zusammensetzung nicht gleich sind. Es geht dies aus folgenden Thatsachen hervor:

1) Ganz anders noch gestalten sich diese Zahlen, wenn der in allen Niederschlägen enthaltene $N$ und daraus Proteinsubstanz mit 18 Prc. $N \cdot$ berechnet wird, ein Verfahren, das dem substantiellen Bestand der Massen zwar nicht entspricht, sich aber zur vergleichenden Úebersicht empfiehlt:

Krystalloidmehl: 1) Extraction mit 2) mit NaCl.-Lös. 3) mit $\mathrm{SO}_{3}$ Kaliwasser.

$\mathrm{N}$ in Säure-Niederschlag $\because \quad .7,163 . \quad 4,118 . \quad 6,20$.

$\mathrm{N}$ in allen übrigen Niederschl. $\quad 3,79 . \quad 4,795 . \quad 2,61$.

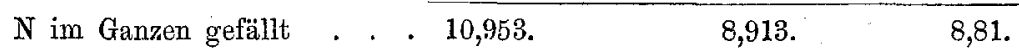

Proteinsubstanz . . . . . 60,79. 49,46. 48,49 Prc.

Nicht gefällter oder ungelöster

$\mathrm{N}$, als Proteinsubst. berechnet $\quad 7,08.18,41 . \quad 18,98$ ” 
U̇eber die Eiweisskörper der Ricinussamen, der Proteinkörner ete. 49

a. Die Menge des Niederschlags nimmt bei anhaltendem Waschen mit Wasser erheblich $a b$;

b. ebenso verhält sich die Cu-Verbindung, die bei langem Waschen mit Wasser an Masse sich um ein bedentendes vermindert;

c. aus der Lösung wird eine wesentlich geringere Menge gefällt, wenn mehr als zur Neutralisation erforderliche Säure angewandt wird und Alkalisalze in einiger Quantität vorhanden sind;

d. der Gehalt des Niederschlags und seiner Cu-Verbindung an $\mathrm{C}$ nimmt bei anbaltendem Waschen mit Wasser ab und ist bei $\mathrm{SO}_{3}$-Füllang geringer als bei der mit Essigsäure (s. pag. 17 und 18,25$)$.

15) Die Zusammensetzung des in den Proteinkörnern und Krystalloiden der Ricinussamen vorwaltenden Eiweisskörpers entspricht derjenigen, welche für die aus mit viel warmem Wasser extrahirten Rückständen von $\mathrm{A}-\mathrm{R}$, und mittelst Fällung durch $\mathrm{H}_{2} \mathrm{O}$ und $\mathrm{CO}_{2}$ aus der NaCl-Lösung von A-R dargestellten Proteinsubstanzen gefunden worden ist und die dem Conglutin aus gelben Lupinen am nächsten steht.

$\begin{array}{ccc}\text { Aus NaCl-Lös. von } & \text { Aus dem Wasser-Extract.- } & \text { Conglutin aus gelben } \\ \text { A-R gefällt. } & \text { Rückst. von A-R. } & \text { Lupinen. } \\ \mathrm{C}=51,31 . & 51,37 . & 50,83 . \\ \mathrm{H}=6,90 . & 6,99 . & 6,92 . \\ \mathrm{N}=18,43 . & 18,14 . & 18,33 . \\ \mathrm{S}=0,97 . & 0,87 . & 0,91 . \\ 0=22,39 . & 22,63 . & 23,04 .\end{array}$

16) Es enthalten jedoch die Proteinkörner sowohl, wie die Krystalloide auch C-reichere und N-ärmere Eiweisskörper; solche wurden gewonnen:

a. Durch Erbitzen der Filtrate von den Niederschlägen nach I; der C-gehalt steigt in dem hierbei entstehenden Coagulationsprodukt auf 53,3 Prc., während der N-Gehalt auf 16,07 Pre. herabsinkt (siehe pag. 18).

b. Durch Zersetzung der Cu-Niederschläge aus denselben Filtraten mittelst angemessener Mengen verdünnter Schwefelsäure, wobei ein Körper mit 53,8 Pre. C und 16,5 Pre. N abgeschieden wird (s. p. 25).

17) Nachgewiesen wurden ferner in Weingeist leicht lösliche und aus dieser Lösung durch absoluten Alkohol fällbare Protein-

E. Pfiñger, Archiv f. Physiologie. Bd. XIX. 
stoffe (Glutenide). Obwohl ihre Zusammensetzung nicht hinreichend genau festgestellt worden ist, ist es doch kaum zweifelhaft, dass sie dem Mucedin und Glutenfibrin am meisten gleichen und die aus dem $\mathrm{Cu}$-Niederschlage wie so eben erwähnt, durch $\mathrm{SO}_{3}$ abgeschiedene Substanz darf der Zusammensetzung nach als eine solche angesehen werden. Sie werden bei dem Verfahren I theilweise gefällt, ein grösserer Theil davon bleibt gelöst; ebenso werden sie bei den übrigen Behandlungsweisen mit Wasser, NaCl-Lösung oder $\mathrm{SO}_{3}$-Wasser in grösserer oder geringerer Menge gelöst, so dass in den Extractionsrückständen nicht bedeutende Reste davon zurückbleiben. Als Bestandtheile von Niederschlägen, deren Masse grösstentheils unlöslich oder schwerlöslich in Wasser ist, werden sie beim Waschen mit Wasser von gewöhnlicher Zimmertemperatur, in welchem sie löslich sind, wenn auch nicht vollständig, so doch der grössten Menge nach abgeschieden.

18) Da reines oder Säure oder Salze enthaltendes Wasser diese Körper gleichzeitig mit dem, dem Conglutin ähnlichen, in gewisser Menge löst und sie aus der wässrigen und NaCl-Lösung durch $\mathrm{CO}_{2}$ oder $\mathrm{H}_{2} \mathrm{O}$ und $\mathrm{CO}_{2}$ mit diesem zum Theil gefällt werden, so erklärt sich hieraus sowohl die geringere Reinheit der aus wässriger Lösung gefällten Substanz, als auch der Unterschied im C-Gehalt der Substanzen aus dem Proteinkörner-reichen Krystalloidmehl und dem Krystalloid-reichen Abschlemmungsriticstande $\mathbf{A}-\mathbf{R}$.

Aus wässriger Lösung 'gefällt.

$$
\begin{array}{cr}
\text { Kryst.-Mehl. } & A-\mathrm{R} . \\
\mathrm{C}=- & 52,34 . \\
\mathrm{H}=- & 6,47 . \\
\mathrm{N}=16,93 . & 17,90 . \\
\mathrm{S}=- & 1,07 . \\
\mathrm{O}=- & 21,95 .
\end{array}
$$

19) Zwischen der mittelst NaCl-Lösung von mir aus Ricinussamen und von Weyl aus Bertholletiafrüchten dargestellten Substanz bestehen so erhebliche Differenzen in der Zasammensetzung, wie oben schon hervorgehoben wurde (p. 46), dass sie nicht für identische Materien angesehen werden können; es folgt hieraus,

a. dass es entweder Proteinstoffe dieser Art von verschiedener Zusammensetzung giebt oder

b. dass die eine von den gefällten Substanzen nicht rein ist, oder 
c. dass bei dieser Darstellungsmethode Zersetzung der ursprïnglichen Substanz eintritt.

Das W eyl' sche sogenannte Pflanzen-Vitellin gleicht in seiner Zusammensetzung so sehr dem Gliadin aus Weizenkleber, was Weyl allerdings nicht bemerkt hat ${ }^{1}$ ), dass man beide für ein und denselben Körper halten könnte, doch ist Gliadin beinahe aschefrei und reicher an $\mathrm{S}$, als das $\mathrm{W}$ eyl'sche Pflanzen-Vitellin. Zur Vergleichung sei hier das Mittel der Analysen (1) und eine EinzelAnalyse eines der reinsten Präparate (2) von Gliadin (s. „Die Eiweisskörper etc." p. 56) angeführt.

Gliadin (aschefrei).

1.

$$
\begin{array}{lc}
\mathrm{C}=52,67 . & 52,40 . \\
\mathrm{H}=7,10 . & 6,92 . \text { (A sche } 0,26) . \\
\mathrm{N}=18,01 . & 18,13 . \\
\mathrm{S}=0,85 . & 0,92 . \\
\mathrm{O}=21,73 . & 21,63 .
\end{array}
$$

Weyl's Pflanzen-Vitellin (aschefrei berechnet).

52,43 .

7,12. (Asche 2,66 =5,36.)

18,10 .

0,55 .

21,88 .

Gleichwohl sind die Körper nicht identisch und offenbar von ganz verschiedener Structur.

Aus den Analysen der Substanzen des Krystalloidmehls und des Abschlemmungs-Rückstandes geht wohl unzweideutig hervor, dass die erstere weniger rein ist in Folge der Lösung und Fällung C-reicherer Proteinstoffe zugleich mit dem Conglutin-ähnlichen Körper. Da Weyl nun nicht reine Paranuss-Krystalloide, sondern ein Gemisch derselben mit Proteinkörnern anwandte, so dürfte die Annahme, dass das Präparat keine reine Substanz darstellt, wohl als zulässig gelten, insbesondere da das Sachssesche Präparat eine der Ricinsubstanz sehr nahestehende Zusammensetzung zeigt.

$$
\begin{array}{cc}
\text { Ricinsubstanz. } & \text { Paranusssubstanz von Sachsse. } \\
\mathrm{C}=51,31 . & 51,42 . \\
\mathrm{H}=6,90 . & 7,31 . \text { (Aschefrei berechnet.) } \\
\mathrm{N}=18,43 . & 18,21 . \\
\mathrm{S}=0,97 . & 1,37 . \\
\mathrm{O}=22,39 . & 21,69 .
\end{array}
$$

1) Wenn Weyl (Hoppe-Seyler, Zeitschr. p. 100) sagt: „Das Vitellin der Paranuss zeichnet sich durch seinen hohen $\mathrm{N}$-Gehalt vor allen bisher bekannten Eiweissstoffen aus", so kann das wohl nicht ernsthaft gemeint sein, da ich meine Untersuchungen über Gliadin und Conglutin schon vor ca. 10 Jahren publicirt habe. 
Die Möglichkeit, dass es verschiedene in den Chloriden von $\mathrm{Na}, \mathrm{K}, \mathrm{NH}_{4}$ und $\mathrm{Ca}$ lösliche und durch $\mathrm{CO}_{2}$ aus der Lösung fällbare Substanzen giebt, oder dass diese überhaupt keine ganz constante Zusammensetzung haben, ist indessen nicht ausgeschlossen.

20) Ueber die Natur der Eiweisskörper in den Ricin-Krystalloiden giebt insbesondere die Untersuchung des AbschlemmungsRückstandes Aufschluss; sie beweist, dass diese Krystalloide in warmem Wasser und in den Lösungen der Chloride von. $\mathrm{K}$, Na, $\mathrm{NH}_{4}$ und $\mathrm{Ca}$ sich wohl vollständig lösen und mindestens 2 Eiweisskörper von verschiedener Zusammensetzung, von denen einer dem Conglutin sehr ähnlich, der andre aber reicher an $\mathrm{C}$ und ärmer an $\mathrm{N}$ ist, darin enthalten sind.

21) Die Proteinkörner enthalten ebenfalls mehrere in Wasser und Chloriden zumeist unlösliche Eiweisskörper, deren einer in der Zusammensetzung dem Conglutin nahekommt, während die ubrigen dem Mucedin oder Glutenfibrin gleichen, in Weingeist löslich sind und mehr $\mathrm{C}$, weniger $\mathrm{N}$ als Conglutin enthalten.

22) Durch Auflösen in Kaliwasser und Fällen mit Säure wird die Zusammensetzung aller dieser Eiweisskörper, die sich leicht darin lösen, nicht geändert.

23) Die aus der Lösung in Kali-haltigem Wasser mit Essigsäu re erhaltenen Fällungen lösen sich in frischem Zustande in $\mathrm{NaCl}$-, KCl- etc. Flüssigkeit theilweise auf, in sehr viel geringerer Menge, wenn mit Schwefelsäure gefällt worden ist.

Die Fällung aus 10 gr A.-R. mittelst Essigsäure gab:

a) Durch $\mathrm{HO}_{2}$ und $\mathrm{CO}_{2}$ aus der $\mathrm{NaCl}-\mathrm{L}$ ös. gefällt.

$0,939 \mathrm{gr}=9,4$ Prc.

Die Fällung vo $0,3426 \mathrm{gr}=1,7$ Prc.

b) In der Fällungstlüssigkeit von a gelöst geblieben. nicht best. c) Unlöslich in NaCl-lösung. 1,784 .

Die Fällung aus einer nicht. gewogenen kleinen Menge zerriebener und entfettetế Paranüsse mit Essigsäure gab:

$0,484 \mathrm{gr}$ nicht best. $0,513 \mathrm{gr}$.

24) Die Proteinsubstanzen der Rieinussamen (Klebermehl und Krystalloide, lösen sich leicht und fast augenblicklich in Kalkund Baryt-Wasser farblos auf. In diesen Lösungen entstehen durch Essigsäure gallertartig-flockige voluminöse Fällungen, die in Chloriden völlig unlöslich sind, wie angenommen werden darf, in Folge der Loslösung der Phosphorsäure von den Eiweisskörpern. Es gaben 
Schtschepotjew: Selbstständige Contraction der Herzspitze etc. $\mathbf{5 3}$

$$
\begin{array}{cc}
\text { 7,5 gr A.-R. } & 7,5 \text { dsgl. } \\
\text { mit Kalkwasser: } & \text { mit Barytwasser: } \\
2,316 \mathrm{gr} & 2,274 \mathrm{gr} \\
=30,9 \text { Pre. } & =30,3 \mathrm{Prc} .
\end{array}
$$

gefällte Proteinsubstanz, während Kaliwasser 32,3 Prc. ergeben hatte. In der Fällungsflüssigheit blieben bedeutende Mengen Proteinsubstanz gelöst.

Die näheren Untersuchungen können in einiger Zeit erst beendet werden.

\section{Selbstständige Contraction der Herzspitze, Veränderungen der Muskeln und der weissen Blut- körperchen unter dem Einfluss von Chinin.}

$$
\text { Von }
$$

Nic. Schtschepotjew.

Zahlreiche, in der medicinischen Litteratur vorhandene, die Wirkung des Chinins auf den thierischen Organismus behandelnde Untersuchungen haben zu folgenden Resultaten geführt: 1) Die Wirkung des Chining auf weisse Blutkörperchen äussert sich in Hemmung oder gänzlicher Aufhebung ihres Vermögens amöboide Bewegungen anszuführen; 2) es wirkt stark auf niedere Organismen ein, verlangsamt oder 'hindert Gährung und Fäulniss; 3) es setzt die Körpertemperatur herab; 4) Herzcontractionen werden von ihm beschleunigt oder verlangsamt (je nach der Dosis); 5) es setzt die Reizbarkeit der Herzmuskelfaser stark herab (Joly et, Le vizki, A, Eụlenburg) ohne eine Einwirikung auf die Skelettmuskeln zu 